\title{
Weak dipole moments of the tau lepton in models with an extended scalar sector
}

\author{
M. A. Arroyo-Ureña and G. Tavares-Velasco \\ Facultad de Ciencias Físico Matemáticas, Benemérita Universidad Autónoma de Puebla, \\ Apartado postal 1152, 72001 Puebla, Puebla, México \\ G. Hernández-Tomé \\ Departamento de Física, CINVESTAV IPN, Apartado Postal 14-740, \\ 07000 México Distrito Federal, México
}

(Received 16 October 2017; published 29 January 2018)

\begin{abstract}
We consider renormalizable couplings of neutral $\phi$, singly $\phi^{ \pm}$, and doubly charged $\phi^{ \pm \pm}$scalar bosons to leptons and the $Z$ gauge boson and calculate the one-loop contributions to the anomalous weak magnetic dipole moment (AWMDM) $a_{\tau}^{W}$ and the weak electric dipole moment (WEDM) $d_{\tau}^{W}$ of a charged lepton in a model-independent way. The analytic expressions are presented in terms of both parametric integrals and Passarino-Veltman scalar functions. Among the new contributions, there are those arising from the vertices of the type $\phi^{ \pm} W^{\mp} Z$ and $Z \phi_{i} \phi_{j}(i \neq j)$, along with contributions from doubly charged scalar bosons. Both $a_{\tau}^{W}$ and $d_{\tau}^{W}$ are evaluated in several scenarios, first in a model-independent way and then within some popular models, such as two-Higgs doublet models (THDMs), multiple-Higgs doublet models and Higgs triplet models. As far as $a_{\tau}^{W}$ is concerned, its real part reaches values as high as $10^{-10}-10^{-9}$ for masses of the new scalar bosons in the $200 \mathrm{GeV}$ range, whereas the imaginary part is 1 or 2 orders of magnitude below. On the other hand, the most promising scenario for a nonvanishing WEDM is offered by a $C P$ violating THDM in a scenario where the heavy neutral scalar bosons are a mixture of $C P$ eigenstates. It is found that the real part of $d_{\tau}^{W}$ is of the order of $10^{-24} \mathrm{ecm}$ and its imaginary part can reach the $10^{-26} \mathrm{ecm}$ level for masses of the new scalar bosons of the order of a few hundred of GeVs. Both the tau AWMDM and WEDM decrease dramatically as the scalar boson masses increase.
\end{abstract}

DOI: 10.1103/PhysRevD.97.013006

\section{INTRODUCTION}

After the 2012 discovery of the Higgs boson by the ATLAS and CMS collaborations at the CERN LHC [1,2], the precise determination of this particle's properties has become one of the most expedited tasks for the experimentalist and so is the search for new physics effects, which may help us to shed light on the yet-unanswered questions of the standard model (SM). The mechanism of spontaneous symmetry breaking (SSB) is achieved in the SM by one complex $S U(2)_{L}$ scalar doublet, thereby leaving as a remnant only one physical Higgs boson. However, there is no compelling reason to expect that this minimal Higgs sector is the one realized in nature. The most simple SM extensions are obtained when one or more scalar multiplets are added to the usual SM Higgs doublet, thereby increasing the spectrum of physical scalar bosons. Therefore,

Published by the American Physical Society under the terms of the Creative Commons Attribution 4.0 International license. Further distribution of this work must maintain attribution to the author(s) and the published article's title, journal citation, and DOI. Funded by SCOAP ${ }^{3}$. models with an extended scalar sector stand out among the most popular and simple SM extensions. A key issue to construct this class of models is to satisfy the $\rho \simeq 1$ relation, along with other theoretical and experimental constraints. It is well known that Higgs multiplet models containing $N$ multiplets with isospin $T_{i}$ and hypercharge $Y_{i}$, whose neutral components develop vacuum expectation values (VEVs) $v_{i}$, modify the tree-level $\rho$ parameter value as follows [3],

$$
\rho=\frac{\sum_{i}^{N} c_{i}\left(T_{i}\left(T_{i}+1\right)-\frac{Y_{i}^{2}}{4}\right) v_{i}^{2}}{\sum_{i}^{N} \frac{Y_{i}^{2} v_{i}^{2}}{2}},
$$

where $c_{i}=1 / 2$ (1) for real (complex) multiplets. Therefore, only those models with an extended scalar sector satisfying the $\rho \simeq 1$ relationship without invoking intricate assumptions are phenomenologically interesting.

Particularly interesting among the models with an extended scalar sector that obey the $\rho=1$ relation at the tree level are Higgs singlet models, two-Higgs doublet models (THDMs), multiple-Higgs doublet models (MHDMs), and Higgs triplet models (HTMs). Apart from their simplicity, this class of models has several motivations: 
new sources of $C P$ violation, the presence of a dark matter candidate, the possibility of accomplishing the seesaw mechanism, the appearance of doubly charged scalar bosons, new tree-level scalar-to-gauge boson couplings, etc. In addition, although these models are interesting on their own, they can be required by other more sophisticated SM extensions, such as the minimal supersymmetric standard model (MSSM), whose scalar sector is a THDM.

New physics effects can be searched for indirectly through virtual corrections from new particles predicted by SM extensions. Along this line, the study of the static electromagnetic properties of fermions provides a unique opportunity to search for this class of effects. The theoretical study of both the anomalous magnetic dipole moment (AMDM) and the electric dipole moment (EDM) of fermions has long received considerable attention, which has been boosted in recent years due to the significant progress in the experimental area. After the study of the electromagnetic properties of a fermion, there has also been great interest in its static weak properties, which are associated with its interaction with the $Z$ gauge boson. The analogues of the AMDM and the EDM are the anomalous weak magnetic dipole moment (AWMDM) $a_{f}^{W}$ and the weak electric dipole moment (WEDM) $d_{f}^{W}$, respectively, which are defined at the $Z$ pole via the dipole terms of the $Z \bar{f} f$ vertex function

$$
\begin{aligned}
i e \bar{u}(p) \Gamma_{Z \bar{f} f}^{\mu}\left(q^{2}\right) u\left(p^{\prime}\right)= & i e \bar{u}(p)\left(F_{2}\left(q^{2}\right) i \sigma^{\mu \nu} q_{\nu}\right. \\
& \left.+F_{3}\left(q^{2}\right) \sigma^{\mu \nu} \gamma_{5} q_{\nu}\right) u\left(p^{\prime}\right),
\end{aligned}
$$

where $q=p-p^{\prime}$ is the $Z$ transfer momentum. The AWMDM is defined as $a_{f}^{W}=-2 m_{f} F_{2}\left(m_{Z}^{2}\right)$ and the WEDM is given by $d_{f}^{W}=-e F_{3}\left(m_{Z}^{2}\right)$. In the SM, $a_{f}^{W}$ arises at the one-loop level and $d_{f}^{W}$ is induced up to the three-loop level [4]. Only the AWMDM and WEDM of heavy fermions are worth studying as those of lighter fermions would be beyond the reach of experimental detection. For instance, in the SM $a_{\tau}^{W}=-(2.10+0.61 i) \times 10^{-6}[5]$ and $d_{\tau}^{W}<8 \times$ $10^{-34} \mathrm{ecm}[6]$. Although the sensitivity reached at the LEP was beyond such a precision level, potentially large contributions from SM extensions can be at the reach of future experiments. The current bounds on the static weak properties of the tau lepton, which were obtained through the study of $\tau^{+} \tau^{-}$production at the LEP by the ALEPH collaboration [7], which used a data sample collected from 1990 to 1995 corresponding to an integrated luminosity of $155 \mathrm{pb}^{-1}$, are shown in Table I. These bounds are well beyond the sensitivity required to test the SM predictions and it is thus worth studying the new physics contributions as they could be large enough to be at the reach of detection in the future.

The AWMDM and WEDM of a fermion have been studied in the context of THDMs [8-10], supersymmetric theories [11,12], unparticles [13], leptoquarks [14], and the simplest little Higgs model [15]. In this work we are interested in analyzing the new contributions arising from
TABLE I. Experimental upper bounds on the static weak properties of the tau lepton [7].

\begin{tabular}{lcc}
\hline \hline & Real part & Imaginary part \\
\hline$a_{\tau}^{W}$ & $1.1 \times 10^{-3}$ & $2.7 \times 10^{-3}$ \\
$d_{\tau}^{W}(\mathrm{ecm})$ & $0.5 \times 10^{-17}$ & $1.1 \times 10^{-17}$ \\
\hline \hline
\end{tabular}

models with an extended scalar sector. We thus calculate the one-loop contributions induced by neutral, singly and doubly charged scalar bosons. Our calculation and numerical analysis will be performed in a model-independent fashion; thereafter the possible implications of some specific models with an extended scalar sector are analyzed. Our results are also useful to compute the contributions arising from the scalar sector of models with an extended gauge sector, which also require additional Higgs multiplets, such as the MSSM, little Higgs models, leftright symmetric models [16], 331 models [17,18], etc.

The rest of this work is organized as follows. A modelindependent calculation of the contribution of new scalar particles to the static weak dipole moments of a charged lepton is presented in Sec. II, whereas the numerical analysis of the contributions to the AWMDM and WEDM of the tau lepton in models with extra scalar multiplets is presented in Sec. III. The conclusions and outlook are presented in Sec. IV. Finally, the necessary Feynman rules and some lengthy formulas for the loop integrals are presented in the appendixes.

\section{NEW SCALAR CONTRIBUTIONS TO THE AWMDM AND WEDM OF CHARGED LEPTONS}

We are interested in the contributions to the AWMDM and WEDM of charged leptons from new neutral, charged and doubly charged scalar particles, which can arise in several models with an extended scalar sector. Our calculation is somewhat general: instead of working out the calculation within a specific model, we consider the scenario of a theory with several nondegenerate neutral, singly and doubly charged scalar bosons with the most general renormalizable couplings to the leptons and the $Z$ gauge boson that can induce the AWMDM and WEDM at the one-loop level. Once the model-independent calculation is presented, we perform the numerical analysis and consider the implications of some extension models.

\section{A. Contributions from new neutral and singly charged scalar bosons}

We first consider lepton number conserving interactions mediated by scalar bosons. For the couplings of a leptonantilepton pair with a neutral or singly charged scalar particle (denoted $\phi_{i}$ or $\phi_{j}$ from now on) we consider the following renormalizable interaction,

$$
\mathcal{L}=i g \bar{\ell}_{l}\left(S_{i l m}+P_{i l m} \gamma_{5}\right) \ell_{m} \phi_{i}+\text { H.c. },
$$


where $\ell_{l}$ is a charged lepton and $\ell_{m}$ is a lepton whose charge depends on that of the scalar boson: if $\phi_{i}$ is a neutral (charged) scalar boson, $\ell_{m}$ is a charged (neutral) lepton. Also, note that we introduce a factor of $g$ for each coupling; we thus expect that the $S_{i l m}$ and $P_{i l m}$ couplings are of the order of $O(1)$ or smaller. Note also that we are considering the most general case where the neutral scalar bosons are a mixture of $C P$-even and $C P$-odd states, which can arise for instance in THDMs with $C P$ violation.

As for the interactions of $Z$ gauge boson with two nondegenerate neutral or charged scalar bosons $\phi_{i}$ and $\phi_{j}$, it is written as follows,

$$
\mathcal{L}=\operatorname{igm}_{Z} g_{Z \phi_{i} \phi_{j}} Z^{\mu} \phi_{i}^{\dagger} \stackrel{\leftrightarrow}{\partial_{\mu}} \phi_{j},
$$

whereas the couplings of the type $Z V \phi_{i}$, with $V$ being a neutral (charged) gauge boson and $\phi_{i}$ a neutral (charged) scalar boson, can be written as

$$
\mathcal{L}=i g g_{\phi_{i} V Z} Z^{\mu} V_{\mu} \phi_{i}+\text { H.c., }
$$

where $V$ stands for a SM gauge boson or another one predicted by a SM extension. Such coupling can be for instance the $\phi_{i} Z Z$ and the $\phi^{ \pm} Z W^{\mp}$ ones. The latter can arise in HTMs at the tree level, whereas in THDMs it arises up to the one-loop level. We also need the interaction between a lepton-antilepton pair with a neutral or charged gauge boson $V$, which we write as

$$
\mathcal{L}=i g \bar{\ell}_{l} \gamma_{\mu}\left(g_{V}^{V l m}-g_{A}^{V l m} \gamma_{5}\right) V^{\mu} \ell_{m}+\text { H.c. }
$$

Particular expressions for the coupling constants $S_{i l m}, P_{i l m}$, $g_{Z \phi_{i} \phi_{i}}$, etc., are known once a specific model is considered. Since we are mainly interested in the contributions arising from models with an extended scalar sector only, we do not consider the contributions of hypothetical gauge bosons or fermions predicted in SM extensions with an extended gauge sector.

The Feynman rules for the above-described couplings are presented in Appendix A. We have used the unitary gauge for our calculation. At the one-loop level, the AWMDM and WEDM of a charged lepton are induced via the Feynman diagrams depicted in Fig. 1, where $\phi_{i}$ and $\phi_{j}$ represent neutral or charged scalar bosons, and $V$ is a gauge boson. Evidently once the electric charge of the scalar bosons is fixed, the charges of the internal lepton $\ell_{m}$ and the gauge boson $V$ also become fixed by charge conservation in each vertex. For instance, if $\phi_{i}$ and $\phi_{j}$ are neutral scalar bosons, $\ell_{m}$ is a charged lepton $e, \mu, \tau$. Thus, for the contributions of new neutral scalar bosons we need the vertices $\phi_{i} \ell_{m} \ell_{l}, \phi_{i} Z Z, Z \phi_{i} \phi_{j}$, and $Z \bar{\ell}_{m} \ell_{m}$. On the other hand, when $\phi_{i}$ and $\phi_{j}$ are charged scalar bosons, the internal lepton is a neutrino $\ell_{m}=\nu_{m}$. Therefore, this class of contributions requires the vertices $\phi_{i}^{+} \bar{\nu}_{m} \ell_{l}, \phi_{i}^{-} W^{+} Z$, $Z \phi_{i}^{-} \phi_{j}^{-}$, and $Z \bar{\nu}_{m} \nu_{m}$.
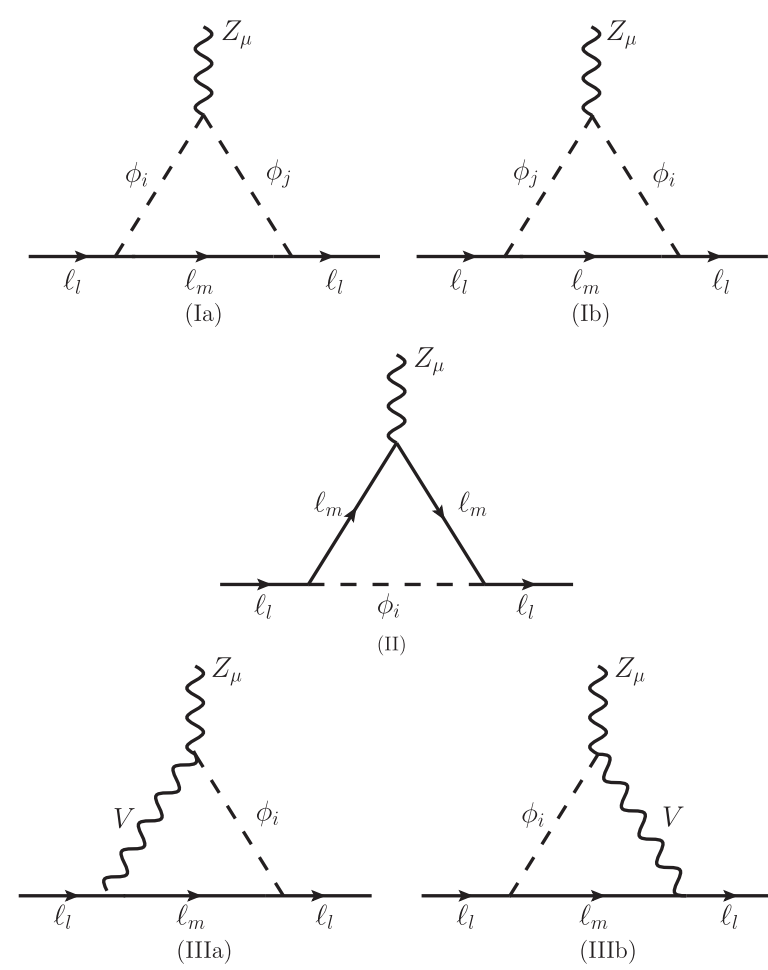

(II)



FIG. 1. Generic Feynman diagrams for the type-I, type-II, and type-III contributions of new neutral and charged scalar bosons to the AWMDM and WEDM of a charged lepton. Here $\ell_{l}$ stands for a charged lepton, whereas $\ell_{m}$ is a lepton whose charge depends on that of the $\phi_{i}$ and $\phi_{j}$ scalar bosons (type-I and type-II diagrams) and that of the $V$ gauge boson and the $\phi_{i}$ scalar boson (type-III diagram).

In order to solve the one-loop integrals we have used both the Feynman parameter technique and the PassarinoVeltman reduction scheme [19], which allows us to crosscheck the results numerically. After some algebra we have obtained the following results.

\section{Anomalous weak magnetic dipole moment}

The contributions to the AWMDM can be written as follows,

$$
\begin{aligned}
a_{l}^{W-I}= & \frac{\alpha \sqrt{x_{l}}}{4 \pi s_{W}^{3}} \sum_{i, j, m} 16 N_{i j} \operatorname{Re}\left[S_{i l m} S_{j l m}^{*} g_{Z \phi_{i} \phi_{j}}^{*}\right] A_{I}^{m \phi_{i} \phi_{j}} \\
& +\left(\begin{array}{c}
\sqrt{x_{m}} \rightarrow-\sqrt{x_{m}} \\
S_{i l m} \rightarrow P_{i l m} \\
S_{j l m} \rightarrow P_{j l m}
\end{array}\right) \\
a_{l}^{W-I I}= & \frac{\alpha \sqrt{x_{l}}}{4 \pi s_{W}^{3}} \sum_{i, m} 16\left(g_{V}^{Z m m}\left\|S_{i l m}\right\|^{2} A_{I I_{1}}^{\phi_{i} m m}\right. \\
& \left.+g_{A}^{Z m m} \sqrt{x_{l}} \operatorname{Re}\left[S_{i l m} P_{i l m}^{*}\right] A_{I I_{2}}^{\phi_{i} m m}\right) \\
& +\left(\begin{array}{c}
\sqrt{x_{m}} \rightarrow-\sqrt{x_{m}} \\
S_{i l m} \leftrightarrow P_{i l m}
\end{array}\right)
\end{aligned}
$$


and

$$
\begin{aligned}
a_{l}^{W-I I I}= & \frac{\alpha \sqrt{x_{l}}}{4 \pi s_{W}^{3}} \sum_{i, m, V} \frac{2 g_{\phi_{i} V Z}}{x_{V}} \operatorname{Re}\left[S_{i l m} g_{V}^{V l m *}\right] A_{I I I}^{m \phi_{i} V} \\
& -\left(\begin{array}{c}
\sqrt{x_{m}} \rightarrow-\sqrt{x_{m}} \\
S_{i l m} \rightarrow P_{i l m} \\
g_{V}^{V l m} \rightarrow g_{A}^{V l m}
\end{array}\right)
\end{aligned}
$$

where $x_{a}=m_{a}^{2} / m_{Z}^{2}, \quad N_{i j}=\left(1-\delta_{i j}\right) \quad$ (1) for neutral (charged) scalar bosons. It is understood that these sums run over all the possible combinations of internal particles predicted by a particular theory. The last term is obtained from the first term after the corresponding replacements are done. As far as the $A_{i}^{A B C}$ functions are concerned, they depend on the masses of the particles circulating into each triangular loop and that of the external lepton (the superscript letters stand for the distinct particles circulating into the loop), but such a dependence has not been written out explicitly to avoid cumbersome equations. The corresponding expressions are presented in Appendix B in terms of both parametric integrals and Passarino-Veltman scalar functions. At this point, it is worth mentioning some important aspects of our calculation. First, we have verified that the contributions to both the AWMDM and WEDM from the diagrams of Fig. 1 are free of ultraviolet divergences. In addition, we have checked that the expressions (7) and (8) reduce to those reported in [20] for the AMDM of a lepton in the limit of $m_{Z} \rightarrow 0$ and after replacing the $Z$ couplings with the photon ones.

\section{Weak electric dipole moment}

As for the contributions to the WEDM, they are given as

$$
\begin{aligned}
& d_{l}^{W-I}= \frac{e \alpha}{4 \pi s_{W}^{3} m_{Z}} \sum_{i, j, m} 4 N_{i j} \operatorname{Im}\left[P_{i l m}^{*} S_{j l m} g_{Z \phi_{i} \phi_{j}}\right] D_{I}^{m \phi_{i} \phi_{j}} \\
&+\left(\begin{array}{c}
\sqrt{x_{m}} \rightarrow-\sqrt{x_{m}} \\
S_{j l m} \rightarrow P_{j l m} \\
P_{i l m} \rightarrow S_{i l m}
\end{array}\right), \\
& d_{l}^{W-I I}=\frac{e \alpha}{4 \pi s_{W}^{3} m_{Z}} \sum_{i, m} 32 g_{V}^{Z m m} \operatorname{Im}\left[S_{i l m} P_{i l m}^{*}\right] D_{I I}^{\phi_{i} m m},
\end{aligned}
$$

and

$$
\begin{aligned}
d_{l}^{W-I I I}= & \frac{e \alpha}{4 \pi s_{W}^{3} m_{Z}} \sum_{i, m, V} \frac{g_{\phi_{i} V Z}}{x_{V}} \operatorname{Im}\left[S_{i l m} g_{A}^{V l m *}\right] D_{I I I}^{m \phi_{i} V} \\
& -\left(\begin{array}{c}
\sqrt{x_{m}} \rightarrow-\sqrt{x_{m}} \\
S_{i l m} \rightarrow P_{i l m} \\
g_{A}^{V l m} \rightarrow g_{V}^{V l m}
\end{array}\right),
\end{aligned}
$$

where again the $D_{i}^{A B C}$ functions are also presented in Appendix $B$ in terms of both parametric integrals and Passarino-Veltman scalar functions.

\section{B. Contribution from doubly charged scalar bosons}

In addition to the above results, we also need to consider the $\Delta L=2$ lepton number violating contributions, which can be mediated by a doubly charged scalar boson. This class of interactions can be written as

$$
\mathcal{L}^{\Delta L=2}=g \ell_{l}^{T} C\left(S_{i l m}^{\prime}+P_{i l m}^{\prime} \gamma^{5}\right) \ell_{m} \phi^{i}+\text { H.c. },
$$

where $C$ is the charge conjugation matrix. Doubly charged scalar bosons can contribute to the AWMDM and WEDM of charged leptons via the Feynman diagrams shown in Fig. 2, where the fermion-flow arrows either clash or emerge from lepton number violating vertices as opposed to lepton number conserving vertices, where the fermion flow follows the same direction. Since we are considering models with an extended scalar sector only, there is no contribution similar to the type-III one of Fig. 1, which requires a doubly charged gauge boson. Some models with extended gauge sector, for instance $S U(3)_{L} \times U(1)_{X}$ models, predict such a particle.

Because of the presence of the charge conjugation matrix and transposed spinors, the Feynman rules for doubly charged scalar bosons must be worked out carefully $[20,21]$. The corresponding Feynman rules and the relevant details for the calculation are presented in Appendix A. After some algebra we have found that the results arising from the Feynman diagrams of Fig. 1 [i.e., Eqs. (7)-(12)] hold true for the contributions of a doubly charged scalar bosons except that a factor of 2 for each lepton number violating vertex must be inserted when the leptons are identical $(l=m)$,

$$
\begin{aligned}
& a_{l}^{W-I^{\prime}}=\left(1+\delta_{l m}\right)^{2} a_{l}^{W-I}, \\
& \left.a_{l}^{W-I I^{\prime}}=1+\delta_{l m}\right)^{2} a_{l}^{W-I I},
\end{aligned}
$$
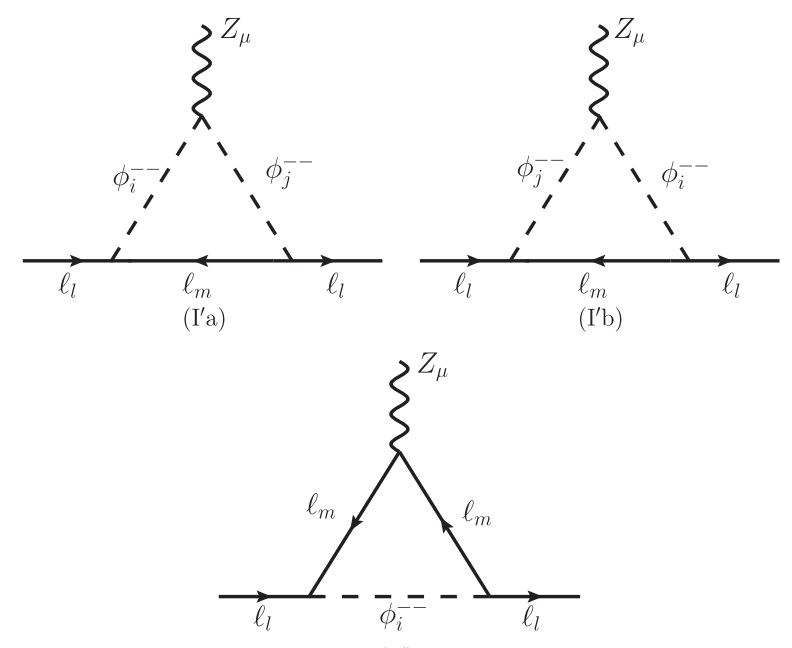

$\left(\mathrm{II}^{\prime}\right)$

FIG. 2. Generic Feynman diagrams for the type- $\mathrm{I}^{\prime}$ and type-II' contributions of doubly charged scalar bosons to the AWMDM and WEDM of charged leptons. Here $\ell_{l}$ and $\ell_{m}$ are both charged leptons. 
where it is understood that one must replace the appropriate couplings and masses involved in each contribution. Similar expressions hold for the contributions to the WEDM arising from lepton number violating vertices. This situation was also noted in the calculation of the AMDM of a lepton [20]. It is worth noting that (9) and (11) would be also valid for a type-III-like contribution arising from a doubly charged scalar and a doubly charged gauge boson, provided that the factor of 2 for vertices with identical leptons is considered and the respective couplings are used.

We now analyze the behavior of the contributions to the AWMDM and WEDM of the tau lepton arising from each type of contribution.

\section{NUMERICAL ANALYSIS}

\section{A. Model-independent analysis of the AWMDM of the tau lepton}

The possible scenarios with new neutral, singly charged, and doubly charged scalar bosons that can induce an AWMDM are summarized in Table II, where we show the corresponding type of Feynman diagram from which such contributions arise and the specific models in which such a scenario is attained. We consider that the neutral scalar bosons are either pure or a mixture of $C P$ eigenstates: $\phi_{i}^{0}$ and $\tilde{\phi}_{i}^{0}$ denote neutral $C P$-even and $C P$-odd scalar bosons, respectively, whereas $\hat{\phi}_{i}^{0}$ stands for a mixture of $C P$ eigenstates. The latter can arise for instance in MHDMs with explicit or spontaneous $C P$ violation in the scalar sector.

As far as neutral scalar bosons are concerned, we note that while a $C P$-even scalar boson can contribute to the AWMDM through type-II and type-III Feynman diagrams, a $C P$-odd scalar boson can only contribute via the type-II diagram. On the other hand, a pair of nondegenerate $\hat{\phi}_{i}^{0}$ and $\hat{\phi}_{j}^{0}$ scalar bosons can induce the AWMDM via the three contributions. As for singly charged scalar bosons, they can contribute via both type-I and type-II contributions, whereas type-III contribution is present only in HTMs, where the $\phi^{ \pm} W^{\mp} Z$ vertex is induced at the tree level. Finally, doubly charged scalar bosons can induce the AWMDM via the type- $\mathrm{I}^{\prime}$ and type- $\mathrm{II}^{\prime}$ diagrams, though type-III-like contributions can also be present in models with a doubly charged gauge boson.

We now proceed to present a numerical analysis of the behavior of the scalar boson contributions to the AWMDM of the tau lepton. Our aim is to examine several scenarios for the contributions of neutral, singly charged, and doubly charged scalar bosons. We first present a model-independent analysis and afterwards we concentrate on some realistic models.

\section{Neutral scalar bosons}

To assess the potential contributions of new neutral scalar bosons to the AWMDM of the tau lepton we consider the minimal scenarios for the each type of contribution to be nonvanishing and estimate the corresponding order of magnitude. The minimal scenarios are as follows:

(i) Type-I contribution requires at least two nondegenerate neutral scalars $\hat{\phi}_{1}$ and $\hat{\phi}_{2}$ that are a mixture of $C P$ eigenstates.

(ii) Type-II contribution requires either a single neutral $C P$-even scalar $\phi_{1}^{0}$ or a single neutral $C P$-odd scalar $\tilde{\phi}_{1}^{0}$.

(iii) Type-III contribution can arise via a single $C P$-even neutral scalar boson $\phi_{1}^{0}$.

Although there could be lepton flavor violating scalar couplings, they are expected to be more suppressed than lepton flavor conserving couplings and we neglect such contributions for simplicity. Therefore, for the internal lepton we take $\ell_{m}=\tau$, whereas $V$ is taken as the $Z$ gauge boson since we are considering that there are no new particles other than extra scalar bosons. For the three minimal scenarios described above we show in Fig. 3 the corresponding contributions to the AWMDM of the tau lepton. For the numerical evaluation we have used the Mathematica numerical routines to evaluate the parametric integrals and a cross-check was done by evaluating the results given in

TABLE II. Contributions to the AWMDM of a charged lepton induced at the one-loop level by the Feynman diagrams of Figs. 1 and 2 arising from new scalar bosons predicted by MHDMs and HTMs. Here $\phi_{i, j}^{0}\left(\tilde{\phi}_{i, j}^{0}\right)$ stand for neutral $C P$-even $(C P$-odd) scalar bosons and $\hat{\phi}_{i, j}^{0}$ for a mixture of $C P$ eigenstates. Note that although the vertex $Z \phi_{i}^{0} \tilde{\phi}_{j}^{0}$ is not forbidden by $C P$ invariance, the type-I contribution to the AWMDM vanishes.

\begin{tabular}{lccc}
\hline \hline Scalar boson(s) & Involved couplings & Type of Feynman diagram & Model \\
\hline$\phi_{i}^{0}$ & $\phi_{i}^{0} \bar{\ell}_{l} \ell_{m}, \phi_{i}^{0} Z Z$ & II-III & MHDMs, HTMs \\
$\tilde{\phi}_{i}^{0}$ & $\tilde{\phi}_{i}^{0} \bar{\ell}_{l} \ell_{m}$ & II & MHDMs, HTMs \\
$\hat{\phi}_{i}^{0}$ and $\hat{\phi}_{j}^{0}$ & $\hat{\phi}_{i, j}^{0} \bar{\ell}_{l} \ell_{m}, Z \hat{\phi}_{i}^{0} \hat{\phi}_{j}^{0}(i \neq j), \hat{\phi}_{i, j}^{0} Z Z$ & I-III & MHDMs \\
$\phi_{i}^{ \pm}$ & $\phi_{i}^{-} \bar{\ell}_{l} \nu_{m}, Z \phi_{i}^{ \pm} \phi_{i}^{\mp}$ & I-II & MHDMs, HTMs \\
$\phi_{i}^{ \pm}$ & $\phi_{i}^{-} \bar{\ell}_{l} \nu_{m}, Z W^{ \pm} \phi_{i, j}^{\mp}$ & III & HTMs \\
$\phi_{i}^{ \pm}$and $\phi_{j}^{ \pm}$ & $\phi_{i, j}^{-} \bar{\ell}_{l} \nu_{m}, Z \phi_{i}^{ \pm} \phi_{j}^{\mp}$ & I-II & MHDMs, HTMs \\
$\phi_{i}^{ \pm \pm}$ & $\phi_{i}^{--} \ell_{l} \ell_{l}, Z \phi_{i}^{ \pm \pm} \phi_{i}^{\mp \mp}$, & $\mathrm{I}^{\prime}-\mathrm{II}$ & HTMs \\
$\phi_{i}^{ \pm \pm}$and $\phi_{j}^{ \pm \pm}$ & $\phi_{i}^{--} \ell_{l} \ell_{l}, Z \phi_{i}^{ \pm \pm} \phi_{j}^{\mp \mp}$, & $\mathrm{I}^{\prime}-\mathrm{II}^{\prime}$ & HTMs \\
\hline \hline
\end{tabular}




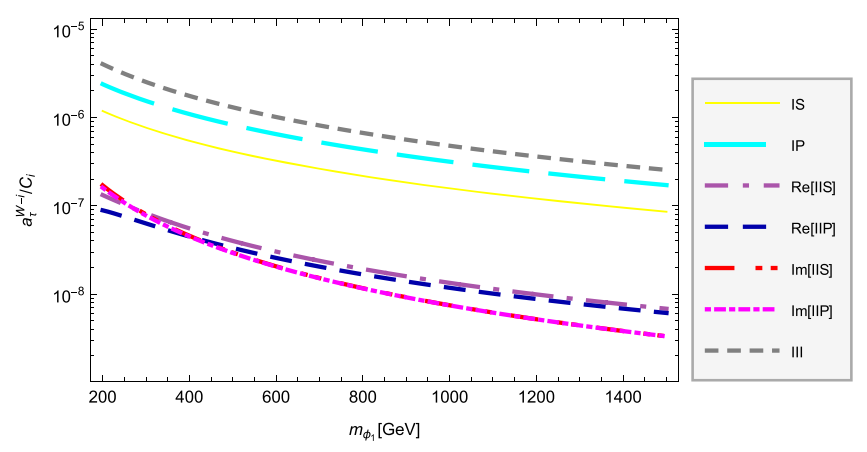

FIG. 3. Absolute values of the contributions from new neutral scalar bosons to the AWMDM of the tau lepton induced by the three types of Feynman diagrams of Fig. 1. Both the real and imaginary parts of type-II contributions are shown. We consider the following scenarios: two nondegenerate scalar bosons $\hat{\phi}_{1}^{0}$ and $\hat{\phi}_{2}^{0}$ with $m_{\hat{\phi}_{1}^{0}}=m_{\phi_{1}}$ and $m_{\hat{\phi}_{2}^{0}}=200 \mathrm{GeV}$ [we take $S_{i \tau \tau} \gg P_{i \tau \tau}$ (IS) and $P_{i \tau \tau} \gg S_{i \tau \tau}$ (IP), for $\left.i=1,2\right]$, a single $C P$-even scalar boson $\phi_{1}^{0}$ (Re[IIS], Im[IIS], and III), and a single $C P$-odd scalar boson $\tilde{\phi}_{1}^{0}(\mathrm{Re}[\mathrm{IIP}]$ and Im[IIP]). In these scenarios each kind of contribution is nearly proportional to the following product of coupling constants: $C_{\mathrm{IS}}=g_{Z \phi_{1} \phi_{2}} \operatorname{Re}\left[S_{1 \tau \tau}^{*} S_{2 \tau \tau}\right], C_{\mathrm{IP}}=$ $g_{Z \phi_{1} \phi_{2}} \operatorname{Re}\left[P_{1 \tau \tau}^{*} P_{2 \tau \tau}\right], C_{\mathrm{IIS}}=\left\|S_{1 \tau \tau}\right\|^{2}, C_{\mathrm{IIP}}=\left\|P_{1 \tau \tau}\right\|^{2}$, and $C_{\mathrm{III}}=$ $g_{\phi_{1} Z Z} \operatorname{Re}\left[S_{1 \tau \tau}\right]$.

terms of Passarino-Veltman scalar functions with the help of the LoopTools routines [22,23]. In the case of the type-I contribution we consider two scenarios: (a) dominant scalar couplings $S_{i \tau \tau} \gg P_{i \tau \tau}$ and (b) dominant pseudoscalar couplings $P_{i \tau \tau} \gg S_{i \tau \tau}$, for $i=1,2$. The type-II contribution is the only one that develops an imaginary part and we show both its real and imaginary parts. Each contribution is nearly proportional to a product of coupling constants denoted by $C_{i}$, as indicated in the caption of the figure.

We observe in the plots of Fig. 3 that the tau AWMDM is highly sensitive to an increase in the scalar boson mass and can get suppressed by about 1 order of magnitude when $m_{\phi_{1}}$ increases from 200 to $1000 \mathrm{GeV}$. As far as the real part of $a_{\tau}^{W}$ is concerned, in the case of type-I contribution, a pair of scalar bosons with scalar couplings larger (smaller) than their pseudoscalar couplings gives a positive (negative) contribution to the AWMDM, but in the case of type-II contribution the $C P$-even $(C P$-odd) scalar boson gives a positive (negative) contribution, which means that there could be cancellations between both contributions. As far as the type-III contribution is concerned, it is always positive and seems to be slightly larger than type-I and type-II contributions. However the values shown in the plots of Fig. 3 could get an additional suppression when the appropriate values for the coupling constants, predicted by a specific model, are inserted. We can thus obtain a rough estimate of the tau AWMDM in a particular model by multiplying the values shown in the plots by the corresponding coupling constants. For instance, if we take either $\left\|S_{\tau \tau}\right\| \sim m_{\tau} /\left(2 m_{W}\right)$ or $\left\|P_{\tau \tau}\right\| \sim m_{\tau} /\left(2 m_{W}\right)$, type-I and
type-II contributions would be suppressed by around 4 orders of magnitude with respect to the values shown in the plot, whereas type-III contribution would be suppressed by 2 orders of magnitude. This is due to the fact that type-III contribution involves only one power of the $S_{\tau \tau}$ or $P_{\tau \tau}$ couplings, whereas both type-I and type-II contributions involve a quadratic power of these couplings, though type-III contribution can have additional suppression due to the $g_{\phi Z Z}$ coupling. In models with several neutral scalar bosons there could be some enhancement provided that the coupling constants are independent and that there is no cancellation between the distinct contributions. However, sum rules between the coupling constants can prevent that all the scalar couplings can be simultaneously large. Also, the presence of scalar bosons that are a mixture of $C P$ eigenstates could not be very relevant for the AWMDM as their contribution gives no considerable enhancement, though the most distinctive signature of this scenario would be the appearance of a WEDM. Finally, the imaginary parts of the type-II contributions to $a_{\tau}^{W}$ from a $C P$-even and $C P$ odd scalar bosons are about the same size but of opposite sign and are 1 order of magnitude smaller than the real part.

\section{Singly charged scalar bosons}

We now turn to focus on the possible contributions from new singly charged scalar bosons, which can arise from the three type of Feynman diagrams of Fig. 1 no matter if there is a lone charged scalar boson. Again we only consider the following minimal scenarios:

(i) Type-I contribution is nonvanishing for a single charged scalar boson, but we consider the scenarios with both a single charged scalar $\phi_{1}^{ \pm}$and two nondegenerate charged scalars $\phi_{1}^{ \pm}$and $\phi_{2}^{ \pm}$.

(ii) Type-II and type-III contributions require a single charged scalar boson $\phi_{1}^{ \pm}$.

The internal lepton $\ell_{m}$ is now a neutrino $\nu_{m}$ and $V$ is the charged $W$ boson. We consider massless neutrinos so there would not be lepton-flavor mixing. In Fig. 4 we show the three types of contributions to the AWMDM of the tau lepton in the scenarios described above. For the charged scalar couplings we assume left-handed couplings, namely, $P_{i \tau \nu_{m}}=-S_{i \tau \nu_{m}}, i=1,2$. Again, the corresponding contributions to the tau AWMDM are nearly proportional to a product of coupling constants, as indicated in the figure. As for the real parts of $a_{\tau}^{W}$, we observe that type-I and type-II contributions are now of similar size and opposite sign, so they can cancel each other out. Thus type-III contribution is expected to be the dominant one among all the contributions of a singly charged scalar, which is worth noting as the $\phi^{\mp} W^{ \pm} Z$ vertex is a peculiarity of HTMs. Note also that typeII contribution is the only one that can develop an imaginary part, which is less than 1 order of magnitude smaller than the real part for $m_{\phi_{1}}=200 \mathrm{GeV}$, but gets considerably suppressed for larger $m_{\phi_{1}}$. Once again, a more careful analysis 


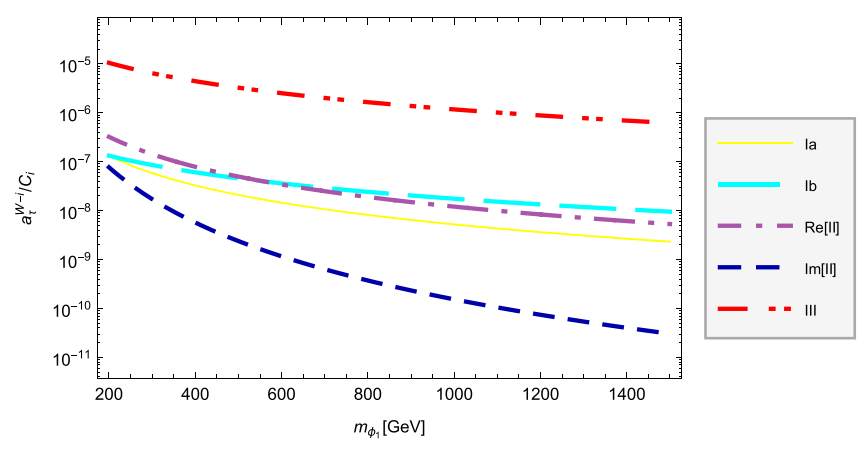

FIG. 4. Absolute values of the contributions from charged scalar bosons to the AWMDM of the tau lepton induced by the three types of Feynman diagrams of Fig. 1. Both the real and imaginary parts of type-II contributions are shown. We consider the following scenarios: two degenerate charged scalar bosons $\phi_{1}^{ \pm}$and $\phi_{2}^{ \pm}$with $m_{\phi_{2}^{ \pm}}=m_{\phi_{1}^{ \pm}}=m_{\phi_{1}}$ (Ia), two nondegenerate charged scalar bosons $\phi_{1}^{ \pm}$and $\phi_{2}^{ \pm}$with $m_{\phi_{2}^{ \pm}}=200 \mathrm{GeV}(\mathrm{Ib})$, and a single charged scalar boson (Re[II], Im[II], and III). We take $P_{i \tau \nu_{m}}=-S_{i \tau \nu_{m}}$ for $i=1$, 2. In these scenarios each kind of contribution is nearly proportional to the following product of coupling constants: $C_{\mathrm{I}}=g_{Z \phi_{1}^{\mp} \phi_{2}^{ \pm}}\left\|S_{\tau \nu_{\tau}}\right\|^{2}, C_{\mathrm{II}}=\left\|S_{\tau \nu_{\tau}}\right\|^{2}$, and $C_{\mathrm{III}}=$ $g_{\phi_{1,2}^{\mp} W^{ \pm} Z} \operatorname{Re}\left[S_{\tau \nu_{\tau}}\right]$.

with appropriate values of the coupling constants is required in a specific model since these contributions can be considerably suppressed as the coupling constants are expected to be much smaller than the unity.

\section{Doubly charged scalar bosons}

Doubly charged scalar bosons can give contributions to the tau lepton AWMDM from type-I' and type-II' Feynman diagrams of Fig. 2. For our analysis we consider the same scenarios as those analyzed in the case of the singly charged scalar boson, except for now absent type-III-like contribution. The internal lepton is now a charged one and for simplicity we assume negligibly lepton flavor violating couplings and take $\ell_{m}=\tau$, in which case there is an extra factor of 4 due to the presence of two vertices with identical leptons. We also consider that the doubly charged scalar boson couplings are left handed, namely, $P_{i \tau \tau}^{\prime}=-S_{i \tau \tau}^{\prime}$, $i=1$, 2. Indeed, doubly charged scalar couplings to charged leptons are left handed (right handed) if they arise from triplets (doublets). We have analyzed the behavior of the AWMDM of the tau lepton induced by doubly charged scalar bosons in scenarios I and II of Fig. 4. For similar masses, the doubly and singly charged scalar contributions only differ by the factor of 4 due to identical leptons. The fact that the internal lepton is now the tau lepton instead of a massless neutrino does not alter significantly these results. Therefore, the results shown in the curves labeled by Ia, Ib, $\operatorname{Re}[\mathrm{II}]$, and $\mathrm{Im}[\mathrm{II}]$ in Fig. 4 are valid for a doubly charged scalar provided that an extra factor of 4 is considered. Also the coupling constants $C_{i}$ should be replaced by
$C_{\mathrm{I}} \rightarrow C_{\mathrm{I}^{\prime}}=g_{{\mathrm{Z \phi _{1 } ^ { \mp \mp }}}^{\mp \pm \pm}{ }_{2}^{ \pm \pm}}\left\|S_{\tau \tau}^{\prime}\right\|^{2} \quad$ and $\quad C_{\mathrm{II}} \rightarrow C_{\mathrm{II}^{\prime}}=\left\|S_{\tau \tau}^{\prime}\right\|^{2}$. Again, since type- $\mathrm{I}^{\prime}$ and type-II' contributions are of opposite sign, there can be large cancellations between them. If there is no type-III-like contribution, doubly charged scalar bosons may induce a more suppressed contribution to the tau AWMDM than neutral and singly charged gauge bosons.

\section{B. AWMDM of the tau lepton in models with an extended scalar sector}

We now turn to present an assessment of the new contributions to the tau AWMDM from some popular models with an extended scalar sector. We only present an overview of each model as they have been largely studied in the literature. For a review of this class of models see [24] and references therein.

\section{Singlet models}

They are the simplest extensions of the SM since in addition to the SM doublet there is only one extra real or complex singlet $S$. Although these models predict new neutral scalars that may play the role of a dark matter candidate and provide a connection with a hypothetical hidden sector (the Higgs portal), their phenomenology is not as interesting as that of models with higher-dimensional multiplets. For instance there are no tree-level flavor change and new sources of $C P$ violation. The interactions of the new physical scalars with the SM particles occur via mixing with the Higgs doublet since the singlet does not couple to the SM fields. Therefore, the new scalar bosons would have suppressed SM-like couplings to the $Z$ gauge boson and the leptons. Furthermore, since at least one of the two new neutral scalar bosons would be a dark matter candidate, the tau AWMDM would receive only new type-II and type-III contributions from one of the new scalar bosons at most, whereas type-I contribution would be absent as it only arises when there is $C P$ violation. We thus conclude that the new contributions to the tau AWMDM from singlet models are not expected to be relevant and we refrain from presenting a more detailed analysis here.

\section{CP-conserving THDMs}

One of the main attractive aspects of THDMs is that they are required by supersymmetric theories, but also can have other interesting features, such as a possible dark matter candidate, flavor change at the tree level, new sources of $C P$ violation, cosmological implications, etc. After SSB, the physical Higgs spectrum of $C P$-conserving THDMs is composed by two $C P$-even neutral scalar bosons $h$ and $H$, one $C P$-odd neutral scalar boson $A$, and a pair of charged scalar bosons $H^{ \pm}$. To forbid tree-level flavor changing neutral currents a $Z_{2}$ symmetry is invoked giving rise to four THDMs with natural flavor conservation according to 
TABLE III. Nonvanishing coupling constants of the new Higgs scalar bosons in the four $C P$-conserving THDMs with natural flavor conservation: THDM of type I (THDM-I), THDM of type II (THDM-II), lepton-specific THDM, and flipped THDM. For the notation of the coupling constants we refer the reader to Fig. 11. The lepton coupling constants must be multiplied by $m_{\tau} /\left(2 m_{W}\right)$. Notice also that the couplings of the flipped THDM (lepton-specific THDM) are the same as those of THDM-I (THDM-II).

\begin{tabular}{lcc}
\hline \hline Coupling & THDM-I & THDM-II \\
\hline$S_{H \tau \tau}$ & $\frac{\sin \alpha}{\sin \beta}$ & $\frac{\cos \alpha}{\cos \beta}$ \\
$P_{A \tau \tau}$ & $-i \cot \beta$ & $i \tan \beta$ \\
$S_{H^{-} \bar{\tau} \nu_{\tau}}$ & $\frac{\cot \beta}{\sqrt{2}}$ & $-\frac{\tan \beta}{\sqrt{2}}$ \\
$P_{H^{-} \nu_{\tau}}$ & $-S_{H^{-} \bar{\tau} \nu_{\tau}}$ & $-S_{H^{-} \bar{\tau} \nu_{\tau}}$ \\
$g_{H Z Z}$ & $\frac{\cos (\alpha-\beta)}{c_{W}}$ & $\frac{\cos (\alpha-\beta)}{c_{W}}$ \\
$g_{Z H^{+} H^{-}}$ & $\frac{1}{2 c_{W}}\left(1-2 s_{W}^{2}\right)$ & $\frac{1}{2 c_{W}}\left(1-2 s_{W}^{2}\right)$ \\
\hline \hline
\end{tabular}

the $Z_{2}$ charge assignments: THDM-I, THDM-II, leptonspecific THDM, and flipped THDM [25]. On the other hand, the so-called THDM-III is obtained by allowing flavor change in the Yukawa Lagrangian and constraining the respective couplings via experimental data. We do not expect a considerable enhancement of the tau AWMDM if flavor violation is allowed, so we only consider flavor conserving models.
$C P$-conserving THDMs would give new type-II contributions to the tau AWMDM arising from the neutral scalar bosons $A$ and $H$. The latter would also give a type-III contribution via the $H Z Z$ vertex, though the $g_{h Z Z}$ and $g_{H Z Z}$ couplings cannot be simultaneously large as they obey the sum rule $g_{h Z Z}^{2}+g_{H Z Z}^{2}=g_{h_{\mathrm{SM}} Z \mathrm{Z}}^{2}$. As for the charged scalar boson $H^{ \pm}$, it would only give type-I and type-II contributions since the $H^{+} W^{-} Z$ vertex is absent at the tree level. The nonvanishing contributions have already been studied in the context of THDM-II, prior to the SM Higgs discovery, [8] and more recently in THDM-III [10]. We calculate the results in both THDM-I and THDM-II considering the most up-to-date bounds on the parameter space. The corresponding Feynman rules have been presented very often in the literature and are summarized in [25]. In Table III we show the couplings of the scalar bosons necessary for our calculation. From now on we consider that $h$ is the SM Higgs boson and its couplings have little deviation from the SM ones.

Constraints on the masses of the new scalar bosons and the parameters $\tan \beta$ and $\alpha$ arise from experimental data. If $h$ is assumed to be the SM Higgs boson, LHC data require $\beta-\alpha \simeq \pi / 2$ (the alignment limit) and small values of $\tan \beta$. In this scenario, the $H Z Z$ vertex would be negligibly small and so would be the corresponding type-III contribution. In Fig. 5 we show the behavior of the real and imaginary parts of the partial and total contributions of model I to the tau AWMDM as functions of the scalar boson masses and two

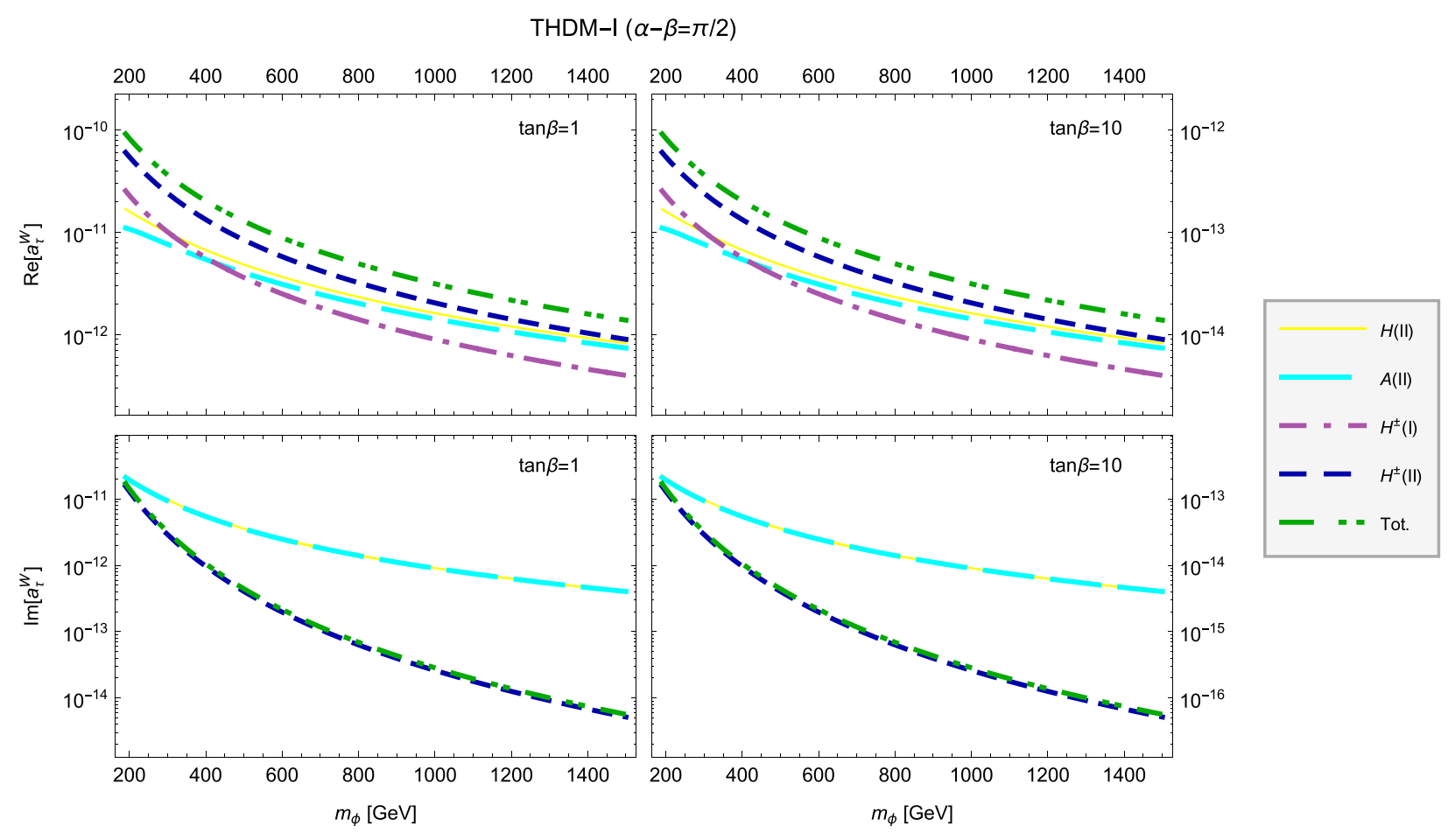

FIG. 5. Absolute values of the real (upper plots) and imaginary (lower plots) parts of the partial and total contributions from the THDM-I to the AWMDM of the tau lepton as functions of the scalar boson masses considering $m_{H}=m_{A}=m_{\phi}$ and the indicated values of the model parameters. The contributions of the flipped THDM are identical. 
$\operatorname{THDM}-\mathrm{II}(\alpha-\beta=\pi / 2)$

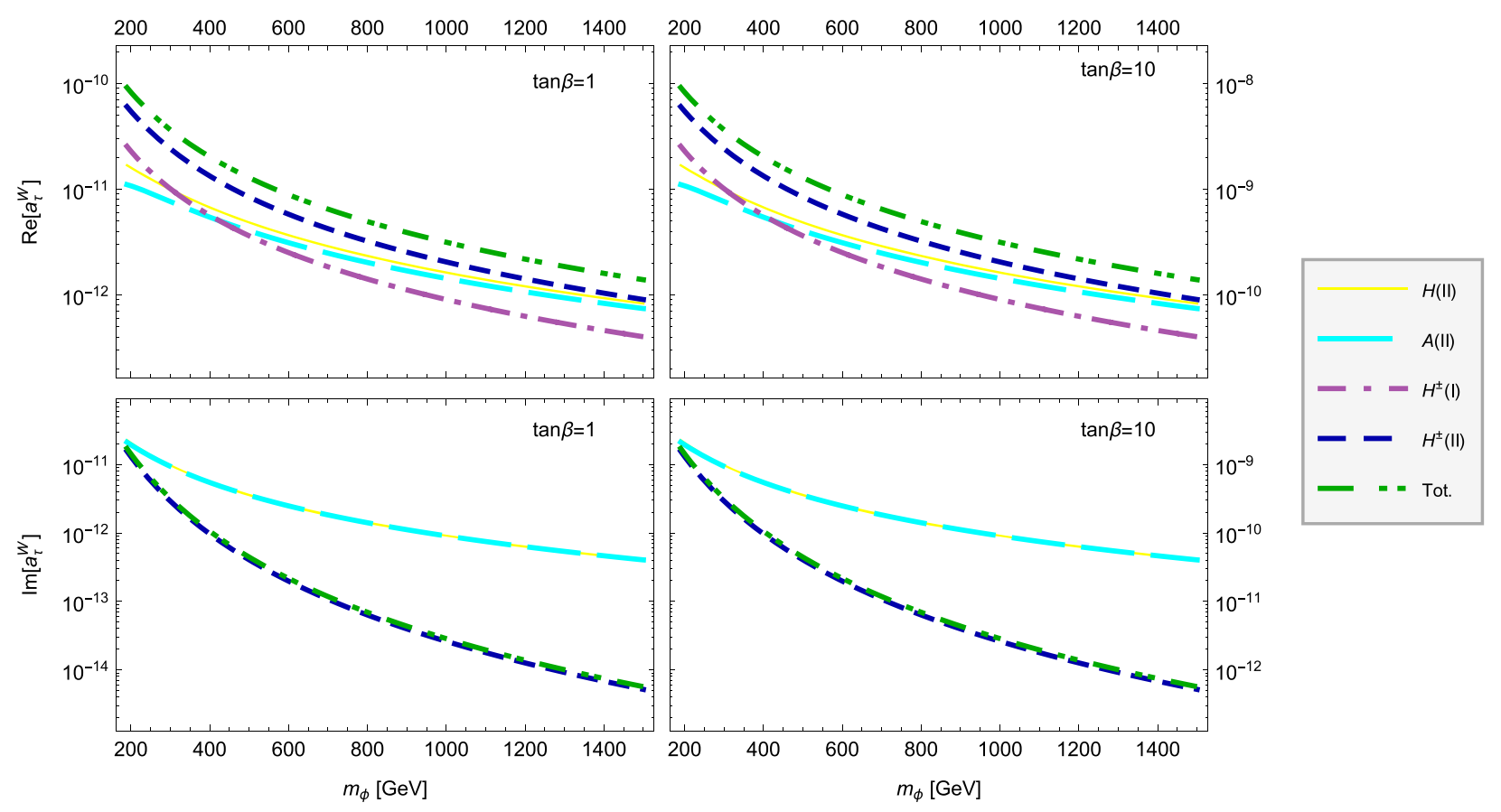

FIG. 6. The same as in Fig. 5 but for THDM-II. These contributions are identical to those of the lepton-specific THDM.

values of $\tan \beta$, whereas in Fig. 6 we present the corresponding plots for the contributions of THDM-II. It turns out that such contributions are identical to those of the flipped THDM and the lepton-specific THDM, respectively. We observe that in THDM-I (THDM-II) all the contribution are proportional to $\cot \beta(\tan \beta)$ in the $\beta-\alpha \simeq$ $\pi / 2$ limit, thus the total contributions are identical in both models for $\tan \beta=1$, but when this parameter increases its value, $a_{\tau}^{W}$ decreases (increases) in THDM-I (THDM-II). The main contributions arise from the charged scalar boson via the type-II diagram, whereas the contributions of the neutral scalar bosons, both of type II, are slightly smaller and of opposite sign. Because of the cancellation between the distinct contributions, the total sum of the real part of $a_{\tau}^{W}$ is of the order of $10^{-10}$ or below for $\tan \beta=1$ and masses of the scalar bosons above the $200 \mathrm{GeV}$ level. On the other hand, when $\tan \beta=10, \operatorname{Re}\left(a_{\tau}^{W}\right)$ is of the order of $10^{-12}$ in THDM-I but of the order of $10^{-8}$ in THDM-II. These values get considerably suppressed as the scalar boson masses increase. As far as the imaginary part of $a_{\tau}^{W}$ is concerned, both the $H$ and $A$ contributions cancel each other out, so the total contribution is due to the charged scalar boson and is of the order of $10^{-11}$ for $m_{H^{ \pm}}=$ $200 \mathrm{GeV}$, but decreases dramatically as $m_{H^{ \pm}}$increases. This contribution is the same in both THDM-I and THDMII for $\tan \beta=1$, but decreases (increases) by 2 orders of magnitude when $\tan \beta=10$ in THDM-I (THDM-II). Although we have focused on two values of $\tan \beta$, we can conclude that the contributions of $C P$-conserving THDMs to the tau AWMDM are much smaller than the pure SM prediction and are even below the contributions of other SM extensions.

\section{CP-violating THDMs}

The most general THDM allows for $C P$ violation in the Higgs sector, which can arise explicitly (via complex couplings) or spontaneously (via complex VEVs). We consider the latter scenario and follow the approach of the authors of Refs. [26,27], where a THDM respecting the $Z_{2}$ symmetry $\Phi_{2} \rightarrow-\Phi_{2}$ and $u_{i R} \rightarrow-u_{i R}$ is considered (as in THDM-II). The most general renormalizable Higgs potential that violates softly the $Z_{2}$ symmetry is given by

$$
V\left(\Phi_{1}, \Phi_{2}\right)=V_{\text {sym }}\left(\Phi_{1}, \Phi_{2}\right)+V_{\text {soft }}\left(\Phi_{1}, \Phi_{2}\right),
$$

where the $Z_{2}$ symmetric term is

$$
\begin{aligned}
V_{\text {sym }}\left(\Phi_{1}, \Phi_{2}\right)= & -\mu_{1}^{2} \Phi_{1}^{\dagger} \Phi_{1}-\mu_{2}^{2} \Phi_{2}^{\dagger} \Phi_{2}+\lambda_{1}\left(\Phi_{1}^{\dagger} \Phi_{1}\right)^{2} \\
& +\lambda_{2}\left(\Phi_{2}^{\dagger} \Phi_{2}\right)^{2}+\lambda_{3}\left(\Phi_{1}^{\dagger} \Phi_{1}\right)\left(\Phi_{2}^{\dagger} \Phi_{2}\right) \\
& +\lambda_{4}\left|\Phi_{1}^{\dagger} \Phi_{2}\right|^{2}+\frac{1}{2}\left(\lambda_{5}\left(\Phi_{1}^{\dagger} \Phi_{2}\right)^{2}+\text { H.c. }\right),
\end{aligned}
$$

whereas the softly violating term is given by

$$
V_{\text {soft }}\left(\Phi_{1}, \Phi_{2}\right)=-\mu_{12}^{2} \Phi_{1}^{\dagger} \Phi_{2}+\text { H.c. }
$$

After SSB $\Phi_{1}$ and $\Phi_{2}$ acquire the VEVs $\Phi_{1}=\left\langle v_{1}\right\rangle / \sqrt{2}$ and $\Phi_{2}^{T}=\left\langle v_{2} e^{i \theta}\right\rangle / \sqrt{2}$ as long as $\left|\mu_{12}^{2} /\left(2 \lambda_{5} v_{1} v_{2}\right)\right|<1$. The three 
neutral physical states $h_{i}$, which are now a mixture of $C P$-even and $C P$-odd eigenstates, are obtained from the gauge eigenstates as follows: $h_{i}=R_{i j} \phi_{j}$, with $h_{i}^{T}=\left(h_{1}, h_{2}, h_{3}\right)$, $\phi^{T}=\sqrt{2}\left(\operatorname{Re}\left(\phi_{1}^{0}\right), \operatorname{Re}\left(\phi_{2}^{0}\right),\left(s_{\beta} \operatorname{Im}\left(\phi_{1}^{0}\right)-c_{\beta} \operatorname{Im}\left(\phi_{2}^{0}\right)\right)\right)$, and $R=$ $R_{3} R_{2} R_{1}$ being a rotation matrix that can be parametrized as

$\mathbf{R}=\left(\begin{array}{ccc}c_{1} & -s_{1} c_{2} & s_{1} s_{2} \\ s_{1} c_{3} & c_{1} c_{2} c_{3}-s_{2} s_{3} & -c_{1} s_{2} c_{3}-c_{2} s_{3} \\ s_{1} s_{3} & c_{1} c_{2} s_{3}+s_{2} c_{3} & -c_{1} s_{2} s_{3}+c_{2} c_{3}\end{array}\right)$,

where $s_{i} \equiv \sin \alpha_{i}$ and $c_{i} \equiv \cos \alpha_{i}$, with $\alpha_{i}$ being the Euler angles $(i=1,2,3)$. The $C P$-conserving THDM-II is obtained in the limit of $\alpha_{2}=\alpha_{3}=0$, after which one must redefine $\alpha_{1}=\pi / 2-\alpha$ to get the conventional nomenclature.

In the Yukawa sector, after SSB the $\ell_{l} \ell_{l} h_{i}$ couplings acquire the form of Eq. (3). As far as the scalar-to-gaugebosons interactions are concerned, there are not only $h_{i} Z Z$ couplings but also $Z h_{i} h_{j}$ ones $(i, j=1,2,3, i \neq j)$, whereas the charged gauge couplings are the same as those of the $C P$-conserving THDM-II. The Feynman rules for this model are presented in [26]. In Table IV we show a summary of the coupling constants required for the evaluation of the tau AWMDM.

In the most general scenario, the tau AWMDM receives contributions from the three neutral scalars via the three types of Feynman diagrams of Fig. 1, whereas the charged scalar boson would contribute through type-I and type-II diagrams. However, it must be noted that the following sum rule is obeyed by the couplings of the scalar bosons to the $Z$ gauge boson [26]: $C_{i}^{2}+C_{j}^{2}+C_{i j}^{2}=1 \quad(i \neq j)$, which means that even though there are additional contributions, some of them would be negligible since not all the coupling constants can increase simultaneously. The properties of the $125 \mathrm{GeV}$ Higgs boson discovered at the LHC seem to fit very well with those of the SM Higgs boson; we

TABLE IV. Nonvanishing coupling constants of the scalar bosons in $C P$-violating THDM-II [26]. For the notation of the coupling constants we refer the reader to Fig. 11. The lepton coupling constants must be multiplied by $m_{\tau} /\left(2 m_{W}\right)$. The $C_{i}$ and $C_{i j}$ constants are $C_{i}=s_{\beta} R_{i 2}+c_{\beta} R_{i 1}$ and $C_{i j}=w_{i} R_{j 3}-w_{j} R_{i 3}$, with $w_{i}=s_{\beta} R_{i 1}-c_{\beta} R_{i 2}$. Note that the couplings of charged scalars remain unchanged.

\begin{tabular}{lc}
\hline \hline Vertex & Coupling constant \\
\hline$S_{h_{i} \tau \tau}$ & $\frac{R_{i 1}}{\cos \beta}$ \\
$P_{h_{i} \tau \tau}$ & $-i \tan \beta R_{i 3}$ \\
$S_{H^{-} \bar{\tau} \nu_{\tau}}$ & $\frac{\tan \beta}{\sqrt{2}}$ \\
$P_{H^{-} \bar{\tau} \nu_{\tau}}$ & $-S_{H^{-} \bar{\tau} \nu_{\tau}}$ \\
$g_{h_{i} Z Z} Z \frac{C}{i}$ & $\frac{C_{W}}{2 c_{W}}$ \\
$g_{Z h_{i} h_{j}}$ & $\frac{1}{2 c_{W}}\left(1-2 s_{W}^{2}\right)$ \\
$g_{Z H^{+} H^{-}}$ & \\
\hline \hline
\end{tabular}

TABLE V. The same as in Table IV but in the scenario with $\alpha_{2}=0$ and $\cos (\alpha-\beta) \simeq 0$. We set $\alpha_{1}=\pi / 2-\alpha$. The lepton coupling constants must be multiplied by $m_{\tau} /\left(2 m_{W}\right)$.

\begin{tabular}{lc}
\hline \hline Vertex & Coupling constant \\
\hline$S_{h_{2} \tau \tau}$ & $-\frac{\cos \alpha \cos \alpha_{3}}{\cos \beta} \simeq \tan \beta \cos \alpha_{3}$ \\
$P_{h_{2} \tau \tau} \operatorname{itan} \beta \sin \alpha_{3}$ \\
$S_{h_{3} \tau \tau}$ & $-\frac{\cos \alpha \sin \alpha_{3}}{\cos \beta} \simeq \tan \beta \sin \alpha_{3}$ \\
$P_{h_{3} \tau \tau}$ & $-i \tan \beta \cos \alpha_{3}$ \\
$g_{\mathrm{Z} h_{2} h_{3}}$ & $-\frac{\sin (\alpha-\beta)}{2 c_{W}}$ \\
\hline \hline
\end{tabular}

thus consider that $h_{1}$ is the lightest scalar boson and its properties are nearly identical to the $125 \mathrm{GeV}$ SM Higgs boson, namely, $C_{1} \simeq 1$. This scenario corresponds to $\alpha_{2} \simeq 0$; therefore $h_{1}$ becomes a pure $C P$-even state and the coupling constants become in this limit $C_{1} \sim C_{23} \sim$ $\sin (\alpha-\beta) \simeq 1$, whereas all of $C_{2}, C_{3}, C_{12}$, and $C_{13}$ become proportional to $\cos (\alpha-\beta) \simeq 0$. The resulting coupling constants are shown in Table V, where we have neglected all terms proportional to $\cos (\alpha-\beta)$. In this case there would only be a new type-I contribution arising from the scalar bosons $h_{2}$ and $h_{3}$, which also would contribute through the type-II diagrams but not the type-III one. In addition, there are also contributions of the charged scalar boson, which are the same as in the $C P$-conserving THDM-II as the charged scalar couplings remain unchanged. In Fig. 7 we show the partial and total contributions to the tau AWMDM for $\tan \beta=1$ and two values of $\sin \alpha_{3}$. We observe that the real part of $a_{\tau}^{W}$ is now dominated by the type-I contribution but it is of the same order of magnitude than the contribution of the $C P$-conserving THDM-II. On the other hand, the imaginary part of $a_{\tau}^{W}$ is dominated by the contributions of the charged scalar boson since the contributions from the neutral scalar bosons cancel each other out. Thus the imaginary part of $a_{\tau}^{W}$ is very similar to that of the $C P$ conserving model. We thus conclude that the contributions of the $C P$-violating THDM give no relevant enhancement to the tau AWMDM as compared to the $C P$-conserving model. However, the most interesting implication of this scenario is the appearance of a WEDM as discussed below.

\section{Multiple Higgs doublet models}

Since the $\rho=1$ relation remains valid at the tree level after the addition of an arbitrary number of Higgs doublets, models with more than two Higgs doublets have also been the focus of considerable attention in the literature, though they are plagued with a large number of free parameters [28]. Apart from their rich phenomenology, MHDMs are particularly appealing as they allow for a plethora of discrete and Abelian symmetries in the scalar and flavor sectors [29-31], which can be useful to reduce the number of free parameters. An $N$-Higgs doublet model predicts $N-1$ pairs of new charged scalar bosons and $2 N-1$ neutral scalar bosons, including the SM Higgs boson. If $C P$ conservation is assumed in the scalar sector, the neutral scalar bosons are 
CPTHDM-II $\left(\tan \beta=1, \alpha_{2}=0, \alpha-\beta=\pi / 2\right)$

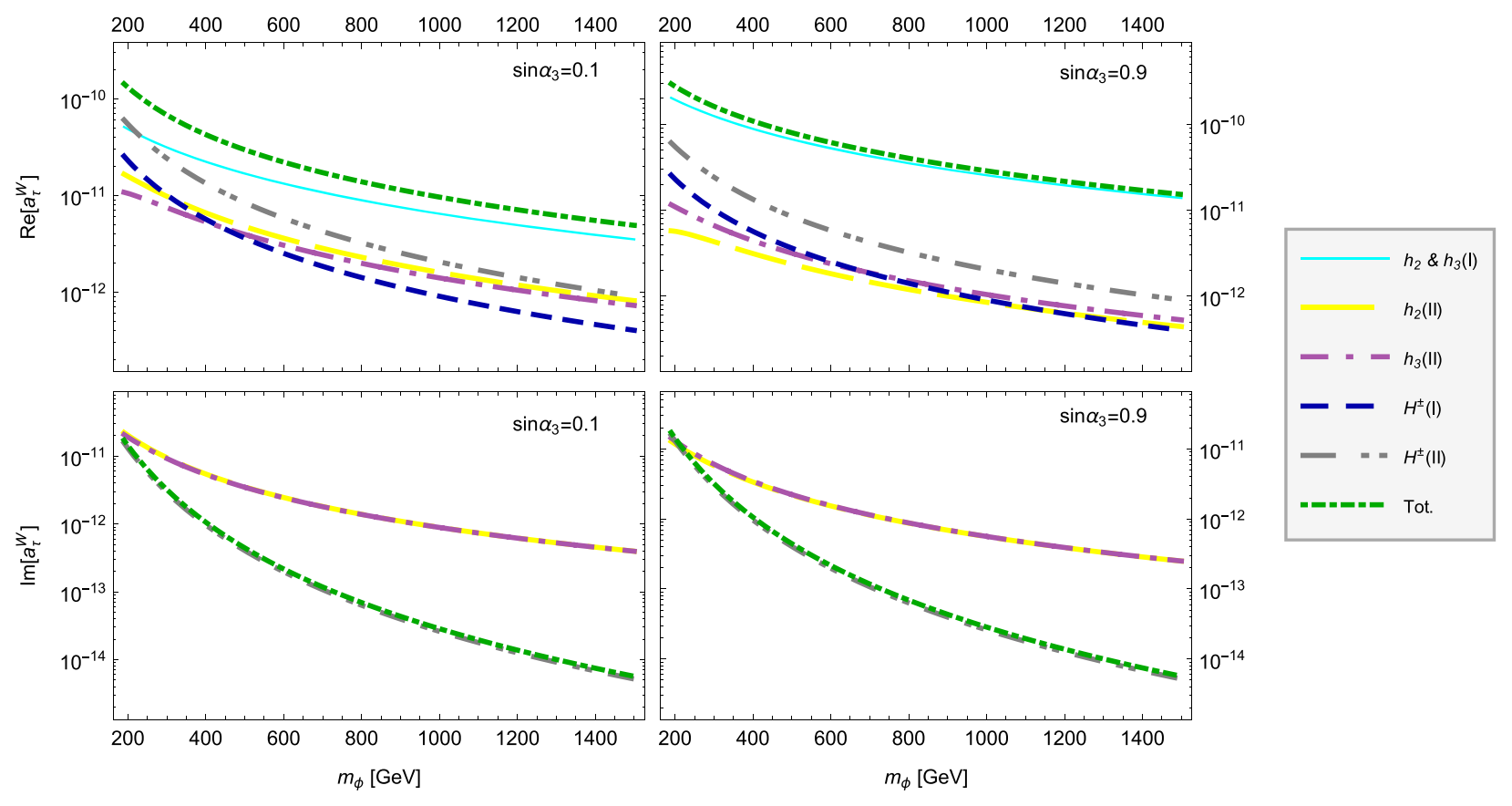

FIG. 7. Absolute values of the real (upper plots) and imaginary (lower plots) parts of the partial and total contributions from the $C P$ violating THDM-II to the AWMDM of the tau lepton as functions of the scalar boson masses considering $m_{h_{1}}=200 \mathrm{GeV}, m_{h_{3}}=m_{\phi}$ and the indicated values of the model parameters.

$C P$ eigenstates, but this is not true when $C P$ violation is allowed. Thus, in principle, there would be more extra contributions to the tau AWMDM. However, a phenomenologically viable MHDM requires that one of the neutral scalar bosons has couplings to the SM particles almost identical to those of the SM Higgs boson. Therefore, it is often assumed that all the neutral Higgs bosons other than the SM-like one are heavy and with suppressed couplings to the SM particles (the decoupling limit). As far as the charged scalar bosons are concerned, their couplings to lepton pairs cannot be simultaneously large due to the corresponding sum rules. It has been pointed out that the more interesting scenario is that in which all the extra charged scalar bosons but only one decouple from the fermions [28]. Therefore, it is not expected that the extra contributions of a MHDM significantly enhance the tau AWMDM as compared to the contribution of a THDM: the extra neutral and charged scalar bosons would be very heavy and with suppressed couplings to the tau lepton and the $Z$ gauge boson.

\section{The Georgi-Machacek model}

Although Higgs triplet models can give dangerous contributions to the $\rho$ parameter, this can be alleviated if either a custodial $S U(2)_{C}$ symmetry is imposed [32,33] or the Higgs triplets VEVs are of the order of a few GeVs [34]. We refer to the former realization as the Georgi-Machacek model (GMM) and to the latter as the Schechter-Valle Higgs triplet model (SVHTM). The most interesting features of these models are the following: naturally light Majorana masses for the neutrinos via the so-called type-II seesaw mechanism, enhanced Higgs-to-gauge boson couplings, doubly charged scalar bosons, and tree-level induced $H^{ \pm} W^{\mp} Z$ coupling [35]. The last two features are an interesting signature of these models, which can offer a rich phenomenology and provide a clear signal at particle colliders. For instance, doubly charged scalars can enhance significantly the two-photon decay of a neutral Higgs boson.

We first discuss the scenario posed by the GMM, which predicts a more rich physical spectrum. This model contains the usual SM doublet $\phi$, one real triplet $\Xi=\left(\xi^{+}, \xi^{0}, \xi^{-}\right)^{T}$ with $Y=0$ and one complex triplet $X=\left(\chi^{++}, \chi^{+}, \chi^{0}\right)^{T}$ with $Y=2$. They are arranged in a bidoublet

$$
\Phi=\left(\begin{array}{cc}
\phi^{0 *} & \phi^{+} \\
-\phi^{+*} & \phi^{0}
\end{array}\right)
$$

and a bitriplet

$$
\Delta(\tilde{X}, \Xi, X)=\left(\begin{array}{ccc}
\chi^{0 *} & \xi^{+} & \chi^{++} \\
-\chi^{+*} & \xi^{0} & \chi^{+} \\
\chi^{++*} & -\xi^{+*} & \chi^{0}
\end{array}\right),
$$

where $\Phi$ and $X$ transform under the custodial symmetry as $\Phi \rightarrow U_{L} \Phi U_{R}^{\dagger}$ and $\Delta \rightarrow U_{L} \Delta U_{R}^{\dagger}$ with $U_{L, R}=e^{\left(i \theta_{L, R}^{a} T^{a}\right)}$. Here $T^{a}=t^{a}$ stands for the $S U(2)$ generators in the triplet representation. To achieve SSB, the neutral components of 
TABLE VI. Coupling constants for vertices inducing the tau AWMDM in the GMM [36]. Here $\theta_{H}$ and $\alpha$ are mixing angles: $\sin \theta_{H}=\sqrt{2} 2 v_{\xi} / v$ and $\alpha$ is the mixing angle of the $h$ and $H$ scalar bosons. For the notation of the coupling constants we refer the reader to Fig. 11. The lepton couplings must be multiplied by $m_{\tau} /\left(2 m_{W}\right)$. Although the $Z H_{3}^{0} h, Z H_{3}^{0} H$, and $Z H_{3}^{0} H_{5}^{0}$ couplings also arise, as explained in the text they do not contribute to the tau AWMDM at the one-loop level.

\begin{tabular}{lc}
\hline \hline Vertex & Coupling constant \\
\hline$S_{H \tau \tau}$ & $\frac{\sin \alpha}{\cos \theta_{H}}$ \\
$P_{H_{3}^{0} \tau \tau}$ & $i \tan \theta_{H}$ \\
$S_{H_{3}^{-} \tau \nu_{\tau}}$ & $\frac{\tan \theta_{H}}{\sqrt{2}}$ \\
$P_{H_{3}^{-} \bar{\tau} \nu_{\tau}}$ & $-S_{H_{3}^{-} \bar{\tau} \nu_{\tau}}^{2}$ \\
$g_{Z H_{3}^{+} H_{3}^{-}}$ & $\frac{1}{2 c_{W}}\left(1-2 s_{W}^{2}\right)$ \\
$g_{H Z Z}$ & $\frac{1}{3 c_{W}}\left(3 c_{H} s_{\alpha}-2 \sqrt{6} s_{H} c_{\alpha}\right)$ \\
\hline \hline
\end{tabular}

the doublet and the triplets acquire VEVs $v_{\phi}, v_{\xi}$, and $v_{\chi}$. It turns out that $\rho=1$ at the tree level since $v_{\xi}$ and $v_{\chi}$ are aligned due to the custodial symmetry. After SSB, nine physical scalar bosons emerge: apart from the SM-like Higgs boson $h$, there are one scalar singlet $H$, one scalar triplet $H_{3}$ $\left(H_{3}^{0}, H_{3}^{ \pm}\right)$, and one scalar fiveplet $H_{5}\left(H_{5}^{0}, H_{5}^{ \pm \pm}, H_{5}^{ \pm}\right)$. While $H$ and $H_{5}^{0}$ are $C P$ even, $H_{3}^{0}$ is $C P$ odd. A peculiarity of this model is that the triplet and fiveplet masses are degenerate. Also, since there is no doublet field in the custodial fiveplet, the $\mathrm{H}_{5}$ states are fermiophobic and their couplings to a fermion antifermion pair can only arise via radiative corrections. The Feynman rules for the GMM are presented in [36]. In Table VI we show the coupling constants necessary to evaluate the tau AWMDM.

In spite of the wide spectrum of physical scalar bosons of the GMM, there is only a handful of new contributions to $a_{\tau}^{W}$ : the $H$ scalar boson would give contributions of type II and type III, $H_{3}^{0}$ would only induce a type-II contribution, and $H_{3}^{ \pm}$would contribute via the type-I and type-II diagrams. It is worth noting that since the fiveplet is fermiophobic, there is no type-III contribution from either the singly charged scalar $H_{5}^{ \pm}$(via the $H_{5}^{ \pm} W^{\mp} Z$ coupling) or the doubly charged scalar $H_{5}^{ \pm \pm}$. Therefore, the contribution of the GMM would be similar to that of a THDM. We consider two sets of values for the $\sin \alpha$ and $\sin \theta_{H}$ parameters still consistent with the constraints on the parameter space of the GMM $[37,38]$ and plot the behavior of the partial and total contributions to the tau AWMDM as functions of the scalar boson masses. The results are shown in Fig. 8, where it is observed that the real part of the type-III contribution arising from the $H$ scalar boson is the dominant one, whereas the real parts of the remaining contributions are considerably suppressed. As for the $a_{\tau}^{W}$ imaginary part, for $\sin \alpha=\sin \theta_{H}=0.1$ the contributions from the $H$ and $H_{3}^{0}$ scalar bosons are nearly identical but of opposite signs; therefore they cancel out and the bulk of the

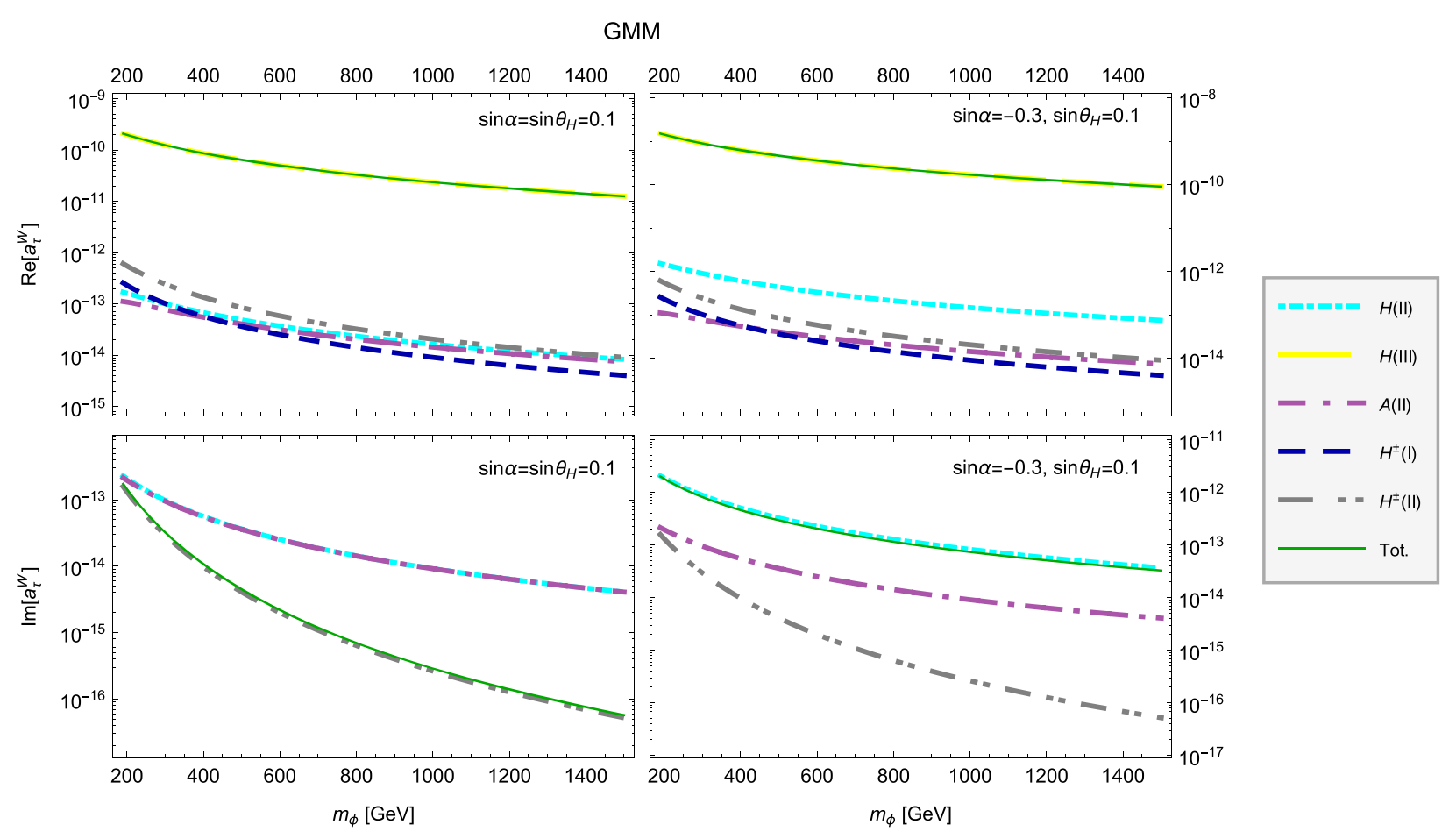

FIG. 8. Absolute values of the real (upper plots) and imaginary (lower plots) parts of the partial and total contributions from the GMM to the AWMDM of the tau lepton as functions of the scalar boson masses considering $m_{H}=m_{H_{3}^{0}}=m_{\phi}$ and the indicated values of the model parameters. 
imaginary part of $a_{\tau}^{W}$ is due to the type-II contribution from the charged scalar boson $H_{3}^{ \pm}$. On the other hand, when $\sin \alpha=-0.3$ and $\sin \theta_{H}=0.1$ the dominant contribution arises from the $H$ scalar boson, whereas the remaining contributions are negligible. In general, the real part of the tau AWMDM can reach the level of $10^{-9}-10^{-10}$ for masses of the new scalar bosons of the order of $200 \mathrm{GeV}$, whereas the imaginary part is of the order of $10^{-12}-10^{-13}$. We observe that the behavior of $a_{\tau}^{W}$ is highly sensitive to the values of $\sin \alpha$ and $\sin \theta_{H}$. It is worth noting that in this model there is no sum rule for the $g_{h Z Z}$ and $g_{H Z Z}$ couplings; thus, the typeIII contribution from the $H$ scalar boson can be relevant for the total contribution. We can conclude that although there is a slight enhancement of $a_{\tau}^{W}$ as compared to the contribution of THDMs, the GMM still gives contributions smaller than those arising from other SM extensions.

\section{The Schechter-Valle Higgs triplet model}

This is another comprehensively studied realization of HTMs [34]. Its main motivation is to generate small neutrino masses via the seesaw mechanism. In such a model only one complex triplet $\Delta$ with $Y=1$ is introduced along with the SM doublet $\Phi$. The triplet is arranged as

$$
\Delta=\left(\begin{array}{cc}
\frac{1}{\sqrt{2}} \delta^{+} & \delta^{++} \\
\delta^{0} & -\frac{1}{\sqrt{2}} \delta^{+}
\end{array}\right) .
$$

The neutrino masses are generated via the following Yukawa Lagrangian,

$$
\mathcal{L}_{Y}=-Y_{\nu i j} L_{i}^{T} C i \sigma_{2} \Delta L_{j}+\text { H.c. }
$$

with $L_{i}^{T}=\left(\nu_{i L}^{T}, e_{i L}^{T}\right)$ and $\mathbf{Y}_{\nu}$ being a symmetric complex matrix.

The Higgs doublet and the neutral component of $\Delta$ acquire VEVs $v_{0}$ and $v_{\Delta}$, with $\sqrt{v_{0}^{2}+v_{\Delta}^{2}}=v=246 \mathrm{GeV}$. To satisfy the $\rho \simeq 1$ constraint the triplet VEV must fulfill $1 \mathrm{eV} \leq v_{\Delta} \leq 8 \mathrm{GeV}$. After SSB, seven physical scalar bosons are left as the remnant: two $C P$-even scalar bosons $H_{1}$ and $H_{2}$, one $C P$-odd scalar boson $A$, a pair of charged scalar bosons $H^{ \pm}$, and a pair of doubly charged scalar bosons $H^{ \pm \pm}$. Other than the doubly charged scalar $H^{ \pm \pm}=\delta^{ \pm \pm}$, the mass eigenstates are mixtures of the doublet $\phi$ and the triplet $\Delta$. The mixing is proportional to $v_{\Delta}$; thus $H_{2}, A$, and $H^{ \pm}$are mainly composed of the triplet fields, whereas $H_{1}$ is predominantly composed of the doublet field and can be identified with the SM Higgs boson. All the extra scalar bosons are nearly mass degenerate. From (23), the following neutrino mass matrix is obtained,

$$
\mathbf{M}_{\nu}=\sqrt{2} v_{\Delta} \mathbf{Y}_{\nu}=\sqrt{2} \mathbf{V}_{\mathrm{PMNS}}^{*} \mathbf{M}_{\nu}^{\mathrm{diag}} \mathbf{V}_{\mathrm{PMNS}}^{\dagger},
$$

TABLE VII. Coupling constants for the vertices inducing the tau AWMDM in the SVHTM [39]. $\boldsymbol{\Gamma}_{+}$and $\boldsymbol{\Gamma}_{++}$are given in (25) and (26). For the notation of the coupling constants we refer the reader to Figs. 11 and 12. The $S_{H_{2} \tau \tau}$ and $P_{A \tau \tau}$ couplings must be multiplied by $m \tau /\left(2 m_{W}\right)$. We used the approximation $v_{0} \simeq v$.

\begin{tabular}{lc}
\hline \hline Vertex & Coupling constant \\
\hline$S_{H_{2} \tau \tau}$ & $-\sin \theta_{0}$ \\
$P_{A \tau \tau}$ & $-i \sin \alpha$ \\
$S_{H^{-} \bar{\ell}_{i} \nu_{j}}$ & $\frac{1}{g} \Gamma_{+}^{i j}$ \\
$P_{H^{-}} \bar{\ell}_{i} \nu_{j}$ & $-S_{H^{-}} \bar{\ell}_{i} \nu_{j}$ \\
$S_{H^{--}} \ell_{i} \ell_{j}$ & $\frac{1}{g} \Gamma_{++}^{i j}$ \\
$P_{H^{--}} \ell_{i} \ell_{j}$ & $-S_{H^{--}} \ell_{i} \ell_{j}$ \\
$g_{H^{+} W^{-} Z}$ & $-S_{W} t_{W}\left(\sin \theta_{+}-\sqrt{2}\left(2+\frac{1}{t_{W}^{2}}\right) \cos \theta_{+} \frac{v_{\Delta}}{v_{0}}\right)$ \\
$g_{H_{2} Z Z}$ & $\sin \theta_{0}-4 \cos \theta_{0} \frac{v_{\Delta}}{v_{0}}$ \\
$g_{Z H^{+} H^{-}}$ & $\frac{1}{2 c_{W}}\left(1-2 s_{W}^{2}\right)$ \\
$g_{Z H^{++} H^{--}}$ & $\frac{1}{c_{W}}\left(1-2 s_{W}^{2}\right)$ \\
\hline \hline
\end{tabular}

with $\mathbf{V}_{\text {PMNS }}$ being the Pontecorvo-Maki-Nakagawa-Sakata mixing matrix.

A more detailed description of this model and the corresponding Feynman rules are presented in Ref. [39]. The coupling constants necessary for the evaluation of $a_{\tau}^{W}$ are shown in Table VII. In particular, the couplings of the charged and doubly charged scalar bosons to leptons are

$$
\boldsymbol{\Gamma}_{+}=\cos \theta_{+} \frac{\mathbf{M}_{\nu}^{\mathrm{diag}} \mathbf{V}_{\mathrm{PMNS}}^{\dagger}}{v_{\Delta}}
$$

and

$$
\boldsymbol{\Gamma}_{++}=\frac{\mathbf{M}_{\nu}}{\sqrt{2} v_{\Delta}} .
$$

Apart from the $H_{1}$ contribution to $a_{\tau}^{W}$, which corresponds to the SM, the new contributions to the tau AWMDM arise from the neutral scalar bosons $\mathrm{H}_{2}$ and $\mathrm{A}$ as well as the singly and doubly charged scalar bosons $H^{ \pm}$ and $H^{ \pm \pm}$. Contrary to the case of the GMM, the charged scalar boson does yield a type-III contribution via the $H^{ \pm} W^{\mp} Z$ vertex. Therefore, in addition to the THDM-like contributions, there are the following additional contributions to the tau AWMDM: a type-III contribution from the singly charged scalar boson and type-I' and type-II' contributions from the doubly charged scalar bosons. However, all these contributions are highly dependent on the value of $v_{\Delta}$ and can be considerably suppressed. Let us first examine the contributions arising from the charged and doubly charged scalar bosons. Since the neutrino mass matrix elements are typically of the order of $10^{-2}-$ $10^{-3} \mathrm{eV}$ [40], for $v_{\Delta}$ of the order of $10^{-5} \mathrm{GeV}$, the matrix elements $\Gamma_{+}^{i j}$ and $\Gamma_{++}^{i j}$ would be of the order of $10^{-6}-10^{-7}$ and even smaller for larger values of $v_{\Delta}$, which means that 
type-I and type-II contributions arising from the charged and doubly charged scalar bosons would be negligibly small, below the $10^{-15}$ level. As far as type-II contributions from the neutral scalar boson are concerned, current constraints from unitarity, the oblique $T$ parameter, and the diphoton strength signal [41] favor the regime where the mixing angles are small and can be approximated as $\sin \theta_{0} \simeq \sin \alpha \simeq 2 v_{\Delta} / v_{0}$ and $\sin \theta_{+} \simeq \sqrt{2} v_{\Delta} / v_{0}$, so the contributions to the tau AWMDM from the neutral scalar bosons are proportional to $\left(v_{\Delta} / v_{0}\right)^{2}$ and so are expected to be very suppressed: for $v_{\Delta}$ around $1 \mathrm{GeV} v_{\Delta} / v_{0} \simeq 8 \times 10^{-3}$; thus the $H_{2}$ and $A$ contributions would be of the order of $10^{-15}$. A similar result is true for type-III contributions arising from the scalar bosons $H_{2}$ and $H^{ \pm}$since all of the $H_{2} Z Z$ and $H^{ \pm} W^{\mp} Z$ coupling constants are proportional to $v_{\Delta} / v_{0}$; thus the respective contribution to $a_{\tau}^{W}$ would be proportional to $\left(v_{\Delta} / v_{0}\right)^{2}$. In conclusion, the extra contributions from the SVHTM to the tau AWMDM would be much smaller than those of THDMs and the GMM.

\section{Models with exotic scalar sectors}

The Georgi-Machacek idea of invoking a $S U(2)$ custodial symmetry to preserve the $\rho=1$ relation at the tree level can be generalized to higher-dimensional multiplets, which is achieved by replacing the Higgs bitriplet by a larger representation under the $S U(2)_{L} \times S U(2)_{R}$ symmetry [42]. These models, dubbed generalized Georgi-Machacek models, have a spectrum of physical scalar bosons composed by the singlet $H$, the triplet $H_{3}$, plus higher fermiophobic multiplets (a fiveplet, a septet, etc.) Therefore, the new contributions to the tau AWMDM would be similar to those of the GMM.

Other models with an exotic scalar sector can be constructed by adding extra higher-dimensional multiplets that respect the relation $\rho=1$ at the tree level. Among such a class of multiplets, the lowest dimensional is a septet with $T=3$ and $Y=4$. A model of this class has been investigated quite recently [43] and it has been dubbed the doublet-septet model. However, the septet does not couple directly to the SM fermions and its interactions only arise through the mixing with the SM doublet. Fifteen physical scalar bosons emerge after SSB, but the relevant ones for the tau AWMDM are two neutral $C P$-even scalars $h$ and $H$, one neutral $C P$-odd scalar $A$, and two pairs of charged scalars $H_{1}^{ \pm}$and $H_{2}^{ \pm}$. Among the remaining physical fields there are a fermiophobic doubly charged scalar and higher-charged scalars. Therefore, apart from the THDM-like contributions, this model only gives a new contribution arising from the extra charged scalar. Thus, we do not expect a substantial increment to the tau AWMDM from the doublet-septet model.

\section{WEDM of the tau lepton}

We now turn to analyze the contributions of new scalar bosons to the tau WEDM, which requires a $C P$-violating phase. Following the same line of discussion as in the
AWMDM case, we only focus on those models with an extended scalar sector; therefore we do not consider additional gauge bosons or fermions. However, there are fewer scenarios for a nonvanishing WEDM than for an AWMDM. For instance, neutral scalar bosons can only induce the tau WEDM at the one-loop level as long as they are a mixture of $C P$ eigenstates. As for a charged scalar boson, type-II contribution vanishes for massless neutrinos, whereas type-I contribution is nonvanishing only for two nondegenerate charged scalar bosons and massive neutrinos. We do not consider the case of doubly charged scalar bosons since, as we have seen above, their couplings to charged leptons are too small to give a relevant contribution to the tau AWMDM, let alone to the WEDM. Another conclusion drawn from our study of the AWMDM is that MHDMs are not expected to give a considerable enhancement to the weak properties of the tau lepton as compared to the contribution of a THDM. Therefore, the most promising scenario for a nonvanishing WEDM of the tau lepton is that posed by a $C P$-violating THDM, so we refrain from analyzing other scenarios. We thus consider the scenario with two nondegenerate neutral scalar bosons that are a mixture of $C P$ eigenstates $\hat{\phi}_{1}$ and $\hat{\phi}_{2}$. The behavior of the tau WEDM as a function of the scalar boson masses is shown in Fig. 9 for some scenarios of interest. Again these contributions could have a strong suppression once the appropriate coupling constants are introduced: for coupling constants of the order of $10^{-2}$, the values shown in the plots would decrease by about 4 orders of magnitude. In addition, stemming from the sum rules obeyed by the coupling constants, some of these contributions would be

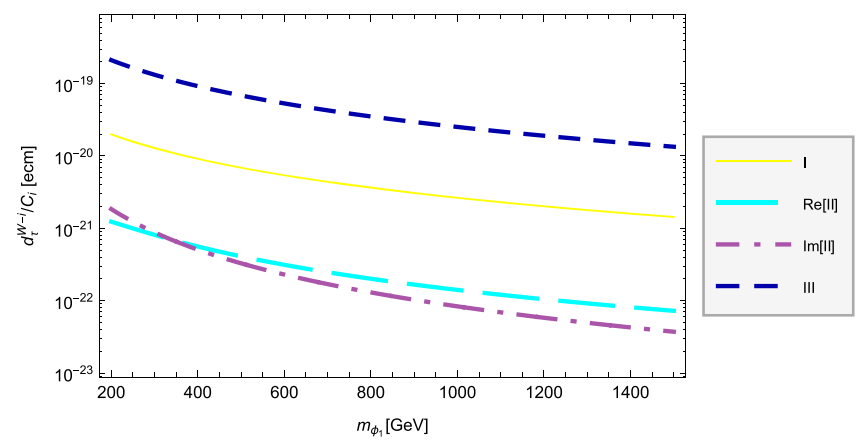

FIG. 9. Absolute values of the contributions from new neutral scalar bosons to the WEDM of the tau lepton induced by the three types of Feynman diagrams of Fig. 1. Both the real and imaginary parts of type-II contributions are shown. We consider the following scenarios: two nondegenerate scalar bosons $\hat{\phi}_{1}^{0}$ and $\hat{\phi}_{2}^{0}$ with $m_{\hat{\phi}_{1}^{0}}=m_{\phi_{1}}$ and $m_{\hat{\phi}_{2}^{0}}=200 \mathrm{GeV}$ (I) as well as a single scalar boson $\hat{\phi}_{1}^{0}(\operatorname{Re}[\mathrm{II}], \mathrm{Im}[\mathrm{II}]$, and III). For type-I contribution we take for simplicity $S_{1 \tau \tau} P_{2 \tau \tau}^{*} \simeq S_{2 \tau \tau} P_{1 \tau \tau}^{*}$, whereas for type-III contribution we use $S_{1 \tau \tau} \gg P_{1 \tau \tau}$. In these scenarios, each kind of contribution is proportional to the following product of coupling constants: $C_{\mathrm{I}}=g_{Z \phi_{1} \phi_{2}} \operatorname{Im}\left[S_{1 \tau \tau} P_{2 \tau \tau}^{*}\right], C_{\mathrm{II}}=\operatorname{Im}\left[S_{1 \tau \tau} P_{1 \tau \tau}^{*}\right]$, and $C_{\mathrm{III}}=$ $g_{\phi_{1} Z Z} \operatorname{Im}\left[S_{1 \tau \tau}\right]$. 


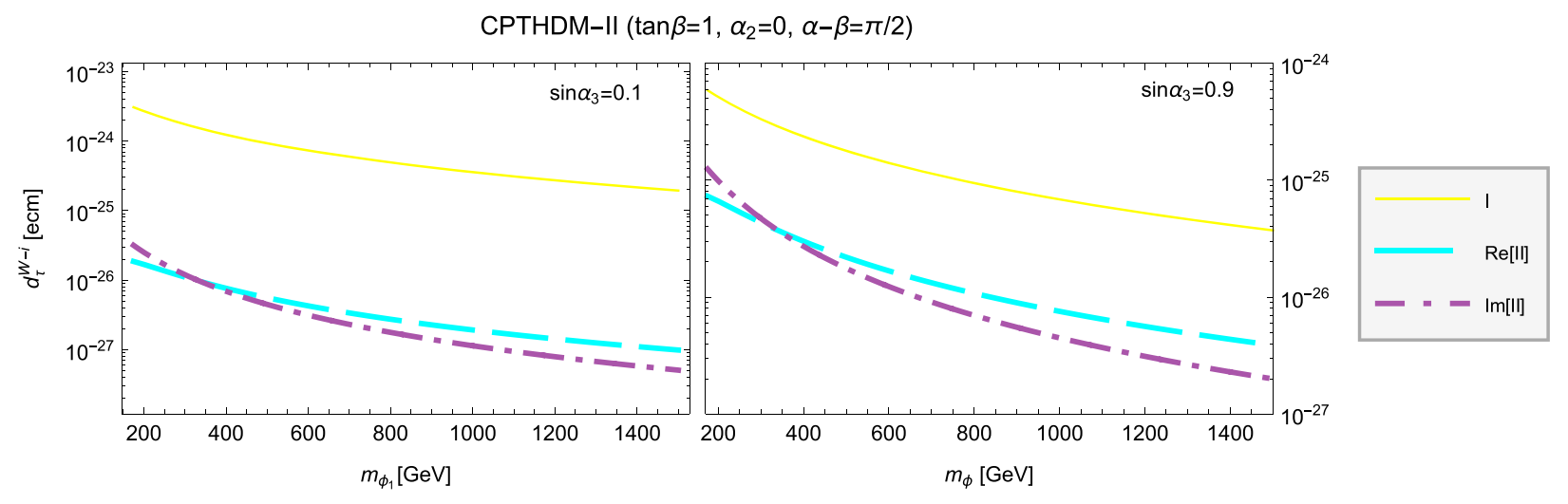

FIG. 10. Absolute values of the real and imaginary parts of the partial contributions from the $C P$-violating THDM-II to the WEDM of the tau lepton as functions of the scalar boson masses considering $m_{h_{2}}=200 \mathrm{GeV}$ and $m_{h_{3}}=m_{\phi}$ for type-I contribution as well as $m_{h_{3}}=m_{h_{2}}=m_{\phi}$ for type-II contribution. We use the indicated values of the model parameters.

additionally suppressed as the accompanying coupling constant could be very small.

Let us now consider the $C P$-violating THDM discussed above [26] and assume the scenario with $\alpha_{2}=0$, in which $h_{1}$ is pure $C P$ even and it thus is identified with the SM Higgs boson, whereas $h_{2}$ and $h_{3}$ are mixtures of $C P$ eigenstates and they would give the three types of contributions to the tau WEDM, though type-III contribution vanishes when $\alpha-\beta=\pi / 2$. We assume massless neutrinos so the contribution of the charged scalar boson vanishes. The corresponding coupling constants are shown in Table IV, where in addition to $\alpha_{2}=0$, we set $\alpha-\beta \simeq$ $\pi / 2$ and use $\alpha_{1}=\pi / 2-\alpha$. We plot the partial contributions to $d_{\tau}^{W}$ from the two neutral scalar bosons $h_{2}$ and $h_{3}$ in Fig. 10 as functions of the scalar boson masses and for two values of $\alpha_{3}$. We can observe that the dominant contribution to $d_{\tau}^{W}$ arises from the Feynman diagram of type I, whereas diagrams of type II give a negligible contribution, which however give the only nonvanishing contribution to the imaginary part of $d_{\tau}^{W}$. In this scenario, the real part of the WEDM of the tau lepton is of the order of $10^{-24} \mathrm{ecm}$ for small $\sin \alpha_{3}$ and decreases by almost 1 order of magnitude for a large $\sin \alpha_{3}$, whereas the imaginary part is of the order of $10^{-26}$ for relatively light masses of the scalar bosons, but decreases quickly as $m_{h_{3}}$ increases.

\section{CONCLUSIONS AND OUTLOOK}

We have performed an analysis of the new one-loop contributions to the tau AWMDM $a_{\tau}^{W}$ and WEDM $d_{\tau}^{W}$ in models with an extended scalar sector, for which we have obtained analytic expressions both in terms of parametric integrals and Passarino-Veltman scalar functions. We first presented a model-independent analysis of the potential contributions of new neutral, singly charged, and doubly charged scalar bosons in several scenarios of interest. Afterwards, we focused on the particular contributions of some specific models.
As far as the contributions to the tau AWMDM arising from new neutral scalar bosons $\phi^{0}$ are concerned, in the most general scenario there can be three types of such contributions (see Figs. 1 and 2) involving the following $Z$ gauge boson couplings: $Z \phi_{i}^{0} \phi_{j}^{0}(i \neq j)$ (type I), $Z \ell \ell$ (type II), and $Z Z \phi^{0}$ (type III). As for type-I contribution, it can only arise in multiple-Higgs doublet models with $C P$ violation. For scalar bosons masses around $200 \mathrm{GeV}$, such a contribution is expected to be of the order of $10^{-10}$ as it is suppressed by two powers of the Yukawa coupling $\phi \ell \ell$. This rough estimate is obtained by assuming that the strength of the $\phi \ell \ell$ coupling is at most of the same order of magnitude as that of the SM Higgs boson. On the other hand, type-II and type-III contributions can arise in multiple-Higgs doublet models and Higgs triplet models even if there is no $C P$ violation. Type-II contribution, which is also suppressed by two powers of the $\phi \ell \ell$ coupling, can reach values of the order of $10^{-11}-10^{-10}$, but it is the only one that can give rise to an imaginary part. On the other hand, type-III contribution may be the largest one as it is suppressed by only one power of the $\phi \ell \ell$ coupling, though an extra suppression can arise from the $Z Z \phi^{0}$ coupling, which can be due to the sum rules obeyed in a specific model. Along this line, it is worth noting that although there can be several partial contributions arising in a particular model, they could cancel each other out instead of adding up. As for the contributions of a singly charged scalar boson $\phi^{ \pm}$, they are induced by the vertices $Z \phi_{i}^{ \pm} \phi_{j}^{\mp}$ (type I), $Z \nu \nu$ (type II), and $Z W^{ \pm} \phi^{\mp}$ (type III). Type-I and type-III contributions, which arise in multiple-Higgs doublet models and Higgs triplet models, can reach values of the order of $10^{-11}-10^{-10}$ for a charged Higgs scalar boson with a mass of the order of $200 \mathrm{GeV}$. Again, type-III contribution may be the largest one, though it can only arise in Higgs triplet models. Finally, a doubly charged scalar boson can contribute only via the vertices $Z \phi^{ \pm \pm} \phi^{\mp \mp}$ (type $\mathrm{I}^{\prime}$ ) and $Z \ell \ell$ (type-II' contribution). These contributions can be of the same order of magnitude as the analogue contributions of singly charged scalar bosons, though there is an additional 
factor of 4 due to the presence of two vertices with identical leptons. We also found that $C P$-violating effects do not significantly alter these predictions; however, they are a necessary ingredient for the presence of a WEDM.

To obtain a more realistic prediction for the tau AWMDM, we considered some popular models with an extended scalar, taking into account the most up-to-date constraints on the corresponding parameter space in view of the LHC data on the SM Higgs boson. We found that twoHiggs doublet models can give the largest contributions to $a_{\tau}^{W}$ arising from two neutral and one singly charged scalar boson. While the real part of $a_{\tau}^{W}$ can reach values as high as $10^{-10}-10^{-9}$ for masses of the new scalar bosons in the $200 \mathrm{GeV}$ range, its imaginary part is 1 or 2 orders of magnitude below. Both contributions decrease quickly as the scalar boson masses increase. Other models such as multiple-Higgs doublet models, the Georgi-Machacek model, and the Schechter-Valle Higgs triplet model can give additional contributions, but they reduce to those of two-Higgs doublet models in the allowed region of parameter space, which stems from the fact that in these models the new scalar bosons typically have suppressed couplings to the SM particles. In particular, doubly charged scalar bosons have very suppressed coupling to the tau lepton in Higgs triplet models. Also, although these models predict the $H^{ \pm} W^{\mp} Z$ vertex at the tree level, its contribution to the tau AWMDM is not relevant. Thus these contributions are expected to be of similar order of magnitude or smaller than those of two-Higgs doublet models.

Contrary to the AWMDM, the scenarios in which a nonvanishing WEDM can arise are very restricted. Although the tau WEDM can receive contributions from all the Feynman diagrams that can induce an AWMDM, it is necessary that the neutral scalar bosons are a mixture of $C P$ eigenstates. We thus considered the scenario with at least two such scalar bosons $\hat{\phi}_{i}$ and performed a model-independent analysis. In this scenario all the types of contributions can arise, including the type-I contribution since there would be a nonvanishing $Z \hat{\phi}_{1} \hat{\phi}_{2}$ vertex. By considering an extra suppression factor of about $10^{-4}$ due to the $\hat{\phi}_{i} \ell \ell$ coupling, we conclude from our analysis that the WEMD of the tau lepton can be in the range of $10^{-25}-10^{-23} \mathrm{ecm}$, with the largest contribution arising from type-III Feynman diagrams. However, as commented above, the size of the $\hat{\phi}_{i} Z Z$ coupling could be very suppressed. When considering specific extension models, we found that the most promising scenario for a nonvanishing WEMD induced by new scalar bosons is that posed by $C P$-violating two-Higgs doublet models, which predict three neutral scalar bosons $h_{i}(i=1$, $2,3)$ that are a mixture of $C P$ eigenstates. However, since one of these scalar bosons must be identified with the SM Higgs boson, current constraints strongly limit the couplings of the two extra neutral scalar bosons. Even more, in this model there are no contributions from the charged scalar boson.
Although other extensions such as Higgs triplet models can also include new sources of $C P$ violation, from our study of the AWMDM we can conclude that the contributions of such models are almost identical to those of a two-Higgs doublet model since any additional contributions are very suppressed. Assuming that $h_{1}$ coincides with the SM Higgs boson, we found that the dominant contributions arise from the neutral scalar bosons $h_{2}$ and $h_{3}$. The real part of $d_{\tau}^{W}$ is of the order of $10^{-24} \mathrm{ecm}$, whereas its imaginary part can reach the $10^{-26} \mathrm{ecm}$ level for masses of the new scalar bosons of the order of a few hundred of GeV.

In summary, the contributions of models with an extended scalar sector to the tau AWMDM and WEDM are smaller than those predicted by other types of extension models. Although interesting on their own, models with an extended scalar sector could be the low-energy approximation of a more fundamental theory still unknown with a strongly interacting ultraviolet completion, which could give an enhancement to the weak dipole moments of a charged lepton. There are several models with extended gauge sector that require a scalar sector with additional scalar multiplets. Among the most popular ones are the MSSM, the left-right $S U(2)_{R} \times S U(2)_{L} \times U(1)_{Y}$ symmetric model and its supersymmetric version, $S U(3)_{L} \times U(1)_{X}$ models, and little Higgs models, to mention a few. These models can include several new contributions to the tau AWMDM and WEDM arising from the new gauge bosons and fermions predicted by these theories. The results presented here can be useful to assess the magnitude of the contribution of the scalar sector of these models. In particular, the contribution to the WEDM can be relevant if there are no additional sources of $C P$ violation from other sector of the model.

\section{ACKNOWLEDGMENTS}

We acknowledge support from Consejo Nacional de Ciencia y Tecnología and Sistema Nacional de Investigadores. Partial support from Vicerrectoría de Investigación y Estudios de Posgrado de la Benemérita Universidad Autónoma de Puebla is also acknowledged. The work of G. H.-T. was supported by Consejo Nacional de Ciencia y Tecnología Grant No. 236394 (Ciencia Básica).

\section{APPENDIX A: FEYNMAN RULES}

In this appendix we present the Feynman rules necessary for the calculation of the static weak properties of a charged lepton. In Fig. 11 we present the generic Feynman rules necessary when lepton number conserving vertices are involved. The fermion, scalar boson, and gauge boson propagators are the usual ones and we refrain from presenting them here.

When lepton number violating vertices mediated by doubly charged scalar bosons are involved, we need the Feynman rules shown in Fig. 12. To simplify the final amplitude we need to exploit the properties of the charge conjugation matrix such as $C=-C^{-1}$ and $C \gamma_{\mu} C^{-1}=\gamma_{\mu}^{T}$. 




FIG. 11. Generic Feynman rules for the lepton number conserving interactions necessary to calculate the weak properties of a charged lepton. Here $\ell_{l}$ stands for a charged lepton and $\ell_{m}$ is either a charged or neutral lepton, whereas the charges of the scalar bosons $\phi_{i, j}$ and the gauge boson $V$ are fixed by charge conservation in each vertex. $\Gamma_{i l m}=S_{i l m}+$ $P_{i l m} \gamma^{5}$ and $\Gamma_{V l m}^{\mu}=\left(g_{V}^{V l m}-g_{A}^{V l m} \gamma^{5}\right) \gamma^{\mu}$.

\section{APPENDIX B: ONE-LOOP FUNCTIONS}

In this appendix we present our results for the $A_{i}^{A B C}$ and $D_{i}^{A B C}$ functions involved in the calculation of the AWMDM and WEDM of a charged lepton presented in Sec. II. We present analytic expressions in terms of both parametric integrals and Passarino-Veltman scalar integrals.

\section{Parametric integrals}

The $A_{i}^{A B C}$ and $D_{i}^{A B C}$ functions can be cast in the form of a one-dimensional parametric integral as follows,

$$
A_{i}^{A B C}=\int_{0}^{1} a_{i}^{A B C}(t) d t
$$

where the letters in the superscript $A B C$ denote the dependence on the masses of the different particles circulating into the loop (in fact $A$ is the particle that couples to

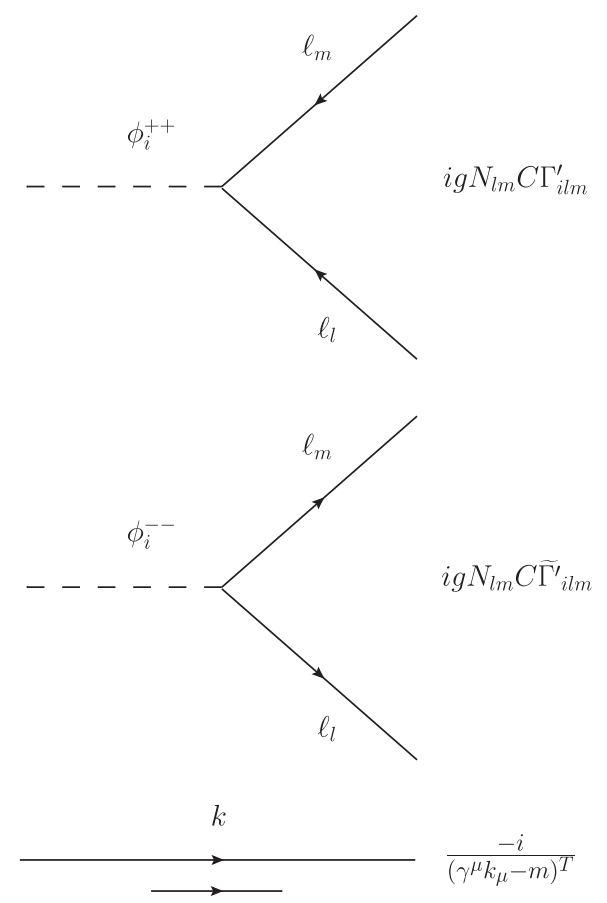

FIG. 12. Generic Feynman rules for the lepton number violating interactions mediated by a doubly charged scalar necessary to calculate the weak properties of a charged lepton. Here $\ell_{l}$ and $\ell_{m}$ are both charged leptons. $C$ is the charge conjugation matrix, $\quad \Gamma_{i l m}^{\prime}=S_{i l m}^{\prime}+P_{i l m}^{\prime} \gamma^{5}, \quad$ and $\quad \tilde{\Gamma}_{i l m}^{\prime}=C \gamma^{0} \Gamma_{i l m}^{\prime \dagger} \gamma^{0} C^{-1}=$ $C\left(S_{i l m}^{\prime *}-P_{i l m}^{\prime *} \gamma^{5}\right) C^{-1} . N_{l m}$ is a symmetry factor that is 2 for $m=$ $l$ and 1 otherwise. Note that the arrow below the fermion propagator stands for the direction in which the Feynman line is read, which in this case coincides with the fermion-flow direction.

both external lepton lines whereas both $B$ and $C$ couple to the $Z$ boson) and the subscript is used to denote distinct functions. Although there is also dependence on the external lepton mass, we omit such a dependence in order to avoid cumbersome expressions; thus we use the shorthand notation $a_{i}^{A B C}(t) \equiv a_{i}^{A B C}\left(t, x_{l}, x_{A}, x_{B}, \ldots\right)$. Similar expressions hold for $D_{i}^{A B C}$ and $d_{i}^{A B C}(t)$.

\section{a. Anomalous weak magnetic dipole moment}

For the type-I Feynman diagram we have

$$
a_{I}^{m \phi_{i} \phi_{j}}(t)=t\left((t-1) \sqrt{x_{l}}-\sqrt{x_{m}}\right) F^{m \phi_{i} \phi_{j}}(t),
$$

with the following auxiliary functions,

$$
\begin{gathered}
F^{A B C}(t)=f^{A B C}(t)+f^{A C B}(t), \\
f^{A B C}(t)=\frac{1}{\xi^{A B C}(t)} \arctan \left[\frac{t-1+x_{B}-x_{C}}{\xi^{A B C}(t)}\right],
\end{gathered}
$$

and

$$
\begin{aligned}
\xi^{A B C}(t)= & {\left[4 t\left((t-1) x_{l}+x_{A}\right)-2(t-1)\left(x_{B}+x_{C}\right)\right.} \\
& \left.-\left(x_{B}-x_{C}\right)^{2}-(1-t)^{2}\right]^{\frac{1}{2}} .
\end{aligned}
$$


As long as the type-II Feynman diagram is concerned, the corresponding $a_{i}^{A B C}$ functions are

$$
a_{I I_{1}}^{\phi_{i} m m}(t)=\left(\sqrt{x_{m}}+t \sqrt{x_{l}}\right)(1-t) F^{\phi_{i} m m}(t),
$$

and

$$
a_{I_{2}}^{\phi_{i} m m}(t)=t(t-1) F^{\phi_{i} m m}(t),
$$

whereas the function associated with the type-III Feynman diagrams, whose amplitudes have been added up, is

$a_{I I I}^{m \phi_{i} V}(t)=f_{0}^{m V}(t)+2 f_{1}^{m \phi_{i} V}(t) F^{m \phi_{i} V}(t)+f_{2}^{\phi_{i} V}(t) G^{m \phi_{i} V}(t)$,

with the auxiliary functions given as

$$
\begin{aligned}
f_{0}^{A B}(t)= & 2(t-1)\left((1-3 t) g^{A B}(t)-2(1-2 t)\right), \\
g^{A B}(t)= & \log \left[t\left((t-1) x_{l}+x_{A}\right)-(t-1) x_{B}\right], \\
G^{A B C}(t)= & g^{A B}(t)-g^{A C}(t), \\
f_{1}^{A B C}(t)= & t^{2}\left(2\left(\sqrt{x_{l}} \sqrt{x_{A}}+8 x_{l}-5 x_{A}\right)+5\left(x_{B}+x_{C}\right)-7\right) \\
& +t\left(4\left(x_{A}-x_{l}\right)-\left(4 x_{C}+7\right) x_{B}\right. \\
& \left.+2 x_{B}^{2}+\left(2 x_{C}-5\right) x_{C}+5\right) \\
& +3 t^{3}\left(1-4 x_{l}\right)-\left(1-x_{B}\right)^{2}+x_{C}^{2},
\end{aligned}
$$

and

$f_{2}^{A B}(t)=t\left(2\left(x_{A}-x_{B}+2\right)-3 t\right)-x_{A}-x_{B}-1$.

\section{b. Weak electric dipole moment}

As far as the contributions to the WEDM of a charged lepton are concerned, they are given through the following functions,

$$
\begin{aligned}
d_{I}^{m \phi_{i} \phi_{j}}(t)= & 2 t\left(\left(x_{\phi_{i}}-x_{\phi_{j}}\right) \sqrt{x_{l}}-\sqrt{x_{m}}\right) F^{m \phi_{i} \phi_{j}}(t) \\
& +t \sqrt{x_{l}} G^{m \phi_{i} \phi_{j}}(t),
\end{aligned}
$$

$$
d_{I I}^{\phi_{i} m m}(t)=\sqrt{x_{m}}(t-1) F^{\phi_{i} m m}(t),
$$

and

$$
\begin{aligned}
d_{I I I}^{m \phi_{i} V}(t)= & -f_{0}^{m V}(t)+2 h_{1}^{m \phi_{i} V}(t) F^{m \phi_{i} V}(t) \\
& +h_{2}^{m \phi_{i} V}(t) G^{m \phi_{i} V}(t),
\end{aligned}
$$

where the $F^{A B C}, G^{A B C}$, and $f^{A B}$ were defined above. The remaining auxiliary functions are

$$
\begin{aligned}
h_{1}^{A B C}(t)= & \left.t^{2}\left(7-18 x_{l}+5\left(2 x_{A}-x_{B}-x_{C}\right)\right)\right) \\
& +t\left(2 x_{C}\left(\sqrt{x_{l}} \sqrt{x_{A}}+x_{l}-x_{C}\right)\right. \\
& +x_{B}\left(2\left(2 x_{C}-\sqrt{x_{l}} \sqrt{x_{A}}-x_{l}\right)+7\right) \\
& \left.+2\left(\sqrt{x_{l}} \sqrt{x_{A}}+3 x_{l}-2 x_{A}-x_{B}^{2}\right)+5\left(x_{C}-1\right)\right) \\
& -3 t^{3}\left(1-4 x_{l}\right)+\left(1-x_{B}\right)^{2}-x_{C}^{2},
\end{aligned}
$$

and

$$
\begin{aligned}
h_{2}^{A B C}(t)= & 1+x_{B}+x_{C}+3 t^{2} \\
& -2 t\left(x_{l}+\sqrt{x_{l}} \sqrt{x_{A}}+x_{B}-x_{C}+2\right) .
\end{aligned}
$$

\section{Passarino-Veltman scalar functions}

We now present our results in terms of PassarinoVeltman scalar functions. We first introduce the following set of ultraviolet finite scalar integrals:

$$
\begin{aligned}
& \Delta_{1}=B_{0}\left(0, m_{A}^{2}, m_{A}^{2}\right)-B_{0}\left(0, m_{B}^{2}, m_{B}^{2}\right), \\
& \Delta_{2}=B_{0}\left(0, m_{B}^{2}, m_{B}^{2}\right)-B_{0}\left(0, m_{C}^{2}, m_{C}^{2}\right), \\
& \Delta_{3}=B_{0}\left(0, m_{C}^{2}, m_{C}^{2}\right)-B_{0}\left(m_{l}^{2}, m_{A}^{2}, m_{B}^{2}\right), \\
& \Delta_{4}=B_{0}\left(m_{l}^{2}, m_{A}^{2}, m_{B}^{2}\right)-B_{0}\left(m_{l}^{2}, m_{A}^{2}, m_{C}^{2}\right), \\
& \Delta_{5}=B_{0}\left(m_{l}^{2}, m_{A}^{2}, m_{C}^{2}\right)-B_{0}\left(m_{Z}^{2}, m_{A}^{2}, m_{B}^{2}\right), \\
& \Delta_{6}=B_{0}\left(m_{l}^{2}, m_{A}^{2}, m_{C}^{2}\right)-B_{0}\left(m_{Z}^{2}, m_{B}^{2}, m_{C}^{2}\right), \\
& \Delta_{7}=m_{Z}^{2} C_{0}\left(m_{l}^{2}, m_{l}^{2}, m_{Z}^{2}, m_{B}^{2}, m_{A}^{2}, m_{C}^{2}\right) .
\end{aligned}
$$

\section{a. Anomalous weak magnetic dipole moment}

The $A_{i}^{A B C}$ functions are given by

$$
\begin{aligned}
A_{I}^{A B C}= & \frac{1}{4 \rho_{l}}\left(x_{B}-2 x_{A}+x_{C}+2 x_{l}-2 x_{A} \Delta_{1}+\left(x_{B}-2 x_{A}\right) \Delta_{2}+\left(x_{B}-2 x_{A}+x_{C}\right) \Delta_{3}-\frac{1}{\delta_{l}}\left(x _ { l } \left(2 \left(x_{A}+\left(2 x_{B}+1\right) x_{l}\right.\right.\right.\right. \\
& \left.\left.\left.+4 \sqrt{x_{A} x_{l}}-2 x_{B}\right)+1\right)-2 \sqrt{x_{A} x_{l}}+x_{A}+x_{C}\left(2 x_{l}\left(1-2 x_{l}\right)-1\right)\right) \Delta_{4}-\frac{2}{\delta_{l}}\left(x_{l}\left(3\left(2 x_{A}-x_{B}-x_{C}\right)+2 x_{l}+1\right)\right. \\
& \left.+2 \sqrt{x_{A} x_{l}}\left(4 x_{l}-1\right)\right) \Delta_{6}-\frac{2}{\delta_{l}}\left(2 x_{l}^{2}\left(2 x_{A}-\left(x_{B}-x_{C}\right)^{2}+x_{B}+x_{C}-1\right)+x_{l}\left(6 x_{A}\left(x_{B}+x_{C}\right)-2 x_{A}\left(3 x_{A}+2\right)\right.\right. \\
& \left.\left.\left.-x_{B}\left(4 x_{C}+x_{B}-1\right)-x_{C}\left(x_{C}-1\right)\right)+\left(1+\left(x_{B}+x_{C}\right)\left(4 x_{l}-1\right)+2 x_{l}\left(4\left(x_{l}-x_{A}\right)-3\right)+2 x_{A}\right) \sqrt{x_{A} x_{l}}+2 x_{l}^{3}\right) \Delta_{7}\right),
\end{aligned}
$$

with $\delta_{l}=1-4 x_{l}$ and $\rho_{l}=\delta_{l} x_{l}$, 


$$
\begin{aligned}
A_{I I_{1}}^{A B C}= & \frac{1}{4 \rho_{l}}\left(x_{B}-x_{A}-x_{l}+x_{B} \Delta_{1}+\left(x_{B}-x_{A}\right) \Delta_{3}-\frac{1}{\delta_{l}}\left(2\left(3 x_{A} x_{l}-\delta_{l} \sqrt{x_{A} x_{l}}\right)-x_{l}\left(6 x_{B}+2 x_{l}+1\right)\right) \Delta_{6}\right. \\
& \left.-\frac{1}{\delta_{l}}\left(2 \delta_{l} \sqrt{x_{A} x_{l}}\left(x_{B}-2 x_{A}+x_{l}\right)-2 x_{l}\left(x_{A}\left(6 x_{B}+2 x_{l}+1-3 x_{A}\right)+\left(x_{l}\left(1-x_{l}-2 x_{B}\right)+x_{B}\left(3 x_{B}+2\right)\right)\right)\right) \Delta_{7}\right), \\
A_{I I_{2}}^{A B C}= & \frac{1}{2 \rho_{l}}\left(x_{A}-x_{B}+x_{l}-x_{B} \Delta_{1}+\left(x_{A}-x_{B}\right) \Delta_{3}-\frac{x_{l}}{\delta_{l}}\left(6\left(x_{B}-x_{A}\right)+2 x_{l}+1\right) \Delta_{6}-\frac{2 x_{l}}{\delta_{l}}\left(\left(2 x_{B}-x_{A}+x_{l}\right)\left(3 x_{A}+x_{l}-1\right)-3 x_{B}^{2}\right) \Delta_{7}\right),
\end{aligned}
$$

and

$$
\begin{aligned}
A_{I I I}^{A B C} & =\frac{1}{\rho_{l}}\left(\left(\sqrt{x_{l}}-\sqrt{x_{A}}\right)\left(\left(2 x_{A}-x_{B}-2 x_{l}-x_{V}\right)+2 x_{A} \Delta_{1}+\left(2 x_{A}-x_{B}\right) \Delta_{2}+\left(2 x_{A}-x_{B}-x_{V}\right) \Delta_{3}\right)\right. \\
& +\frac{1}{\delta_{l}}\left(\left(\sqrt{x_{l}}-\sqrt{x_{A}}\right)\left(x_{A}-2 \delta_{l} \sqrt{x_{A} x_{l}}+x_{l}\left(2 x_{A}+\left(4 x_{B}\left(x_{l}-1\right)+2 x_{l}+1\right)\right)\right)-x_{V}\left(\sqrt{x_{A}}\left(2 x_{l}\left(1-2 x_{l}\right)-1\right)\right.\right. \\
& \left.\left.+\sqrt{x_{l}}\left(2 x_{l}\left(3-14 x_{l}\right)+1\right)\right)\right) \Delta_{4}+\frac{2 \sqrt{x_{l}}}{\delta_{l}}\left(x_{l}\left(2\left(x_{A}+x_{l}\right)+3 x_{B}-5 x_{V}-1\right)+3 \sqrt{x_{A} x_{l}}\left(x_{B}-2 x_{A}+x_{V}+2 x_{l}-1\right)\right. \\
& \left.+2\left(x_{A}-x_{V}\right)\right) \Delta_{6}+\frac{2 \sqrt{x_{l}}}{\delta_{l}}\left(x_{V}\left(2 x_{B}-3 x_{A}+x_{l}\left(6\left(2 x_{B}+1\right) x_{l}-8 x_{B}+3\left(2 x_{A}-1\right)\right)\right)+x_{A}\left(2 x_{l}\left(x_{B}+1-2 x_{l}\right)\right.\right. \\
& \left.+x_{B}-1+2 x_{A}\left(x_{l}-1\right)\right)+\sqrt{x_{A} x_{l}}\left(\left(x_{B}-1\right)^{2}+2 x_{V}\left(x_{V}\left(x_{l}+1\right)+\left(1-2 x_{B}\right)\left(x_{l}-1\right)+3 x_{A}\right)+6 x_{A}\left(x_{A}+\left(1-x_{B}-2 x_{l}\right)\right)\right. \\
& \left.\left.\left.+2 x_{l}\left(x_{B}\left(x_{B}+1\right)-2+3 x_{l}\right)\right)+x_{l}\left(x_{B}\left(1-x_{B}\right)\left(2 x_{l}+1\right)+2 x_{l}\left(x_{l}-1\right)+x_{V}^{2}\left(14 x_{l}-5\right)\right)\right) \Delta_{7}\right) .
\end{aligned}
$$

\section{b. Weak electric dipole moment}

As for the $D_{i}^{A B C}$ functions, they are given by

$$
\begin{aligned}
D_{I}^{A B C}= & \frac{2}{\rho_{l}}\left(\frac{1}{2} \delta_{l}^{2} x_{B}\left(x_{B}-x_{C}\right) \Delta_{2}+\delta_{l}\left(x_{B}-x_{C}\right) \Delta_{3}-\left(x_{l}\left(4 x_{A}+2 x_{B}+\delta_{l}-6 x_{C}\right)+2 \sqrt{x_{A} x_{l}}+x_{C}-x_{A}\right) \Delta_{4}\right. \\
& -4\left(\sqrt{x_{A} x_{l}}+x_{l}\left(x_{B}-x_{C}\right)\right) \Delta_{5}+2\left(\left(\sqrt{x_{A} x_{l}}+x_{l}\left(x_{B}-x_{C}\right)\right)\left(1+2 x_{A}-x_{B}-x_{C}\right)\right. \\
& \left.\left.-2 x_{l}\left(\sqrt{x_{A} x_{l}}-x_{l}\left(x_{C}-x_{B}\right)\right)\right) \Delta_{7}\right), \\
D_{I I}^{A B C}= & \frac{16 \sqrt{x_{A} x_{l}}}{\rho_{l}}\left(-\Delta_{6}+\left(x_{B}-x_{A}+x_{l}\right) \Delta_{7}\right),
\end{aligned}
$$

and

$$
\begin{aligned}
D_{I I I}^{A B C}= & \frac{1}{\rho_{l} x_{V}}\left(\delta_{l}\left(\sqrt{x_{A}}+\sqrt{x_{l}}\right)\left(x_{V}-x_{B}\right)\right)-x_{B} \delta_{l}\left(\sqrt{x_{A}}+\sqrt{x_{l}}\right) \Delta_{2}+\delta_{l}\left(\sqrt{x_{A}}+\sqrt{x_{l}}\right)\left(x_{V}-x_{B}\right) \Delta_{3}+\left(x _ { V } \left(\sqrt{x_{A}}\left(1-6 x_{l}\right)\right.\right. \\
& \left.\left.+\sqrt{x_{l}}\left(2 x_{l}+1\right)\right)+\left(\sqrt{x_{A}}+\sqrt{x_{l}}\right)\left(x_{l}\left(4 x_{A}+2 x_{B}+1-4 x_{l}\right)-2 \sqrt{x_{A} x_{l}}-x_{A}\right)\right) \Delta_{4}-2 \sqrt{x_{l}}\left(\sqrt{x_{A} x_{l}}\left(x_{V}-x_{B}+1\right)\right. \\
& \left.+x_{A}-x_{V}+x_{l}\left(x_{V}-x_{B}\right)\right) \Delta_{6}+2 \sqrt{x_{l}}\left(x _ { B } \left(2\left(x_{V}-\sqrt{x_{A} x_{l}}\left(1+x_{A}-x_{l}\right)\right)-x_{l}\left(2\left(x_{A}+2 x_{V}-x_{l}\right)+1\right)\right.\right. \\
& \left.-x_{A}+x_{B}\left(\sqrt{x_{A} x_{l}}+x_{l}\right)\right)+\sqrt{x_{A} x_{l}}\left(x_{V}+1\right)\left(2\left(x_{A}-x_{l}\right)-x_{V}+1\right)+x_{l}\left(2 x_{A}\left(x_{V}-1\right)+x_{V}\left(3 x_{V}-1-2 x_{l}\right)\right) \\
& \left.\left.+x_{A}\left(2 x_{A}-3 x_{V}+1\right)\right) \Delta_{7}\right) .
\end{aligned}
$$


[1] G. Aad et al. (ATLAS Collaboration), Phys. Lett. B 716, 1 (2012).

[2] S. Chatrchyan et al. (CMS Collaboration), Phys. Lett. B 716, 30 (2012).

[3] J. F. Gunion, H. E. Haber, G. L. Kane, and S. Dawson, The Higgs Hunter's Guide 80, 1 (Addison-Wesley, Reading, MA, 1989).

[4] M. J. Booth, arXiv:hep-ph/9301293.

[5] J. Bernabeu, G. A. Gonzalez-Sprinberg, M. Tung, and J. Vidal, Nucl. Phys. B436, 474 (1995).

[6] W. Bernreuther, U. Low, J. P. Ma, and O. Nachtmann, Z. Phys. C 43, 117 (1989).

[7] A. Heister et al. (ALEPH Collaboration), Eur. Phys. J. C 30, 291 (2003).

[8] J. Bernabeu, D. Comelli, L. Lavoura, and J. P. Silva, Phys. Rev. D 53, 5222 (1996).

[9] D. Gomez-Dumm and G. A. Gonzalez-Sprinberg, Eur. Phys. J. C 11, 293 (1999).

[10] M. Arroyo-Ureña and E. Díaz, J. Phys. G 43, 045002 (2016).

[11] W. Hollik, J. I. Illana, C. Schappacher, D. Stockinger, and S. Rigolin, Nucl. Phys. B557, 407 (1999).

[12] W. Hollik, J. I. Illana, S. Rigolin, C. Schappacher, and D. Stockinger, Nucl. Phys. B551, 3 (1999).

[13] A. Moyotl and G. Tavares-Velasco, Phys. Rev. D 86, 013014 (2012).

[14] A. Bolaños, A. Moyotl, and G. Tavares-Velasco, Phys. Rev. D 89, 055025 (2014).

[15] M. A. Arroyo-Ureña, G. Hernández-Tomé, and G. Tavares-Velasco, Eur. Phys. J. C 77, 227 (2017).

[16] R. N. Mohapatra and J. C. Pati, Phys. Rev. D 11, 2558 (1975).

[17] F. Pisano and V. Pleitez, Phys. Rev. D 46, 410 (1992).

[18] P. H. Frampton, Phys. Rev. Lett. 69, 2889 (1992).

[19] G. Passarino and M. J. G. Veltman, Nucl. Phys. B160, 151 (1979).

[20] S. Moore, K. Whisnant, and B.-L. Young, Phys. Rev. D 31, 105 (1985).

[21] A. Denner, H. Eck, O. Hahn, and J. Kublbeck, Nucl. Phys. B387, 467 (1992).
[22] G. J. van Oldenborgh and J. A. M. Vermaseren, Z. Phys. C 46, 425 (1990).

[23] T. Hahn and M. Perez-Victoria, Comput. Phys. Commun. 118, 153 (1999).

[24] I. P. Ivanov, Prog. Part. Nucl. Phys. 95, 160 (2017).

[25] G. C. Branco, P. M. Ferreira, L. Lavoura, M. N. Rebelo, M. Sher, and J. P. Silva, Phys. Rep. 516, 1 (2012).

[26] J. F. Gunion, B. Grzadkowski, H. E. Haber, and J. Kalinowski, Phys. Rev. Lett. 79, 982 (1997).

[27] B. Grzadkowski, J. F. Gunion, and J. Kalinowski, Phys. Rev. D 60, 075011 (1999).

[28] Y. Grossman, Nucl. Phys. B426, 355 (1994).

[29] I. P. Ivanov, V. Keus, and E. Vdovin, J. Phys. A 45, 215201 (2012).

[30] I. P. Ivanov and E. Vdovin, Phys. Rev. D 86, 095030 (2012).

[31] I. P. Ivanov and C. C. Nishi, J. High Energy Phys. 01 (2015) 021.

[32] H. Georgi and M. Machacek, Nucl. Phys. B262, 463 (1985).

[33] M. S. Chanowitz and M. Golden, Phys. Lett. B 165, 105 (1985).

[34] J. Schechter and J. W. F. Valle, Phys. Rev. D 22, 2227 (1980).

[35] S. Kraml, et al., arXiv:hep-ph/0608079.

[36] C.-W. Chiang and K. Yagyu, J. High Energy Phys. 01 (2013) 026.

[37] K. Hartling, K. Kumar, and H. E. Logan, Phys. Rev. D 91, 015013 (2015).

[38] C.-W. Chiang, S. Kanemura, and K. Yagyu, Phys. Rev. D 90, 115025 (2014).

[39] P. Fileviez Perez, T. Han, G.-y. Huang, T. Li, and K. Wang, Phys. Rev. D 78, 015018 (2008).

[40] C. Patrignani et al. (Particle Data Group), Chin. Phys. C 40, 100001 (2016).

[41] D. Das and A. Santamaria, Phys. Rev. D 94, 015015 (2016).

[42] H. E. Logan and V. Rentala, Phys. Rev. D 92, 075011 (2015).

[43] C. Alvarado, L. Lehman, and B. Ostdiek, J. High Energy Phys. 05 (2014) 150. 